\title{
Fritillariae Thunbergii Bulbus: Traditional Uses, Phytochemistry, Pharmacodynamics, Pharmacokinetics and Toxicity
}

\author{
Hong $\mathrm{Li}^{1}{ }^{1}$, Andrew Hung ${ }^{2}$, Mingdi Li ${ }^{1}$ and Angela Wei Hong Yang ${ }^{1, *}$ \\ 1 School of Health and Biomedical Sciences, RMIT University, Bundoora, Victoria 3083, Australia; \\ s3640112@student.rmit.edu.au (H.L.); s3335658@student.rmit.edu.au (M.L.) \\ 2 School of Science, RMIT University, Melbourne, Victoria 3001, Australia; andrew.hung@rmit.edu.au \\ * Correspondence: angela.yang@rmit.edu.au; Tel.: +61-3-9925-7175; Fax: +61-3-9925-7178
}

Received: 15 March 2019; Accepted: 30 March 2019; Published: 3 April 2019

\begin{abstract}
Fritillariae Thunbergii Bulbus (FTB) has been widely used as an antitussive herb for thousands of years in China. However, FTB's traditional uses, chemical compounds and pharmacological activities have not been systematically reviewed. This study aimed to review its traditional uses, phytochemistry, pharmacodynamics, pharmacokinetics and toxicity. We searched the Encyclopedia of Traditional Chinese Medicine to explore the historical records which indicate that it acts to clear heat, resolve phlegm, relieve cough, remove toxicity and disperse abscesses and nodules. We searched 11 databases to identify potential phytochemical or pharmacological studies. Characteristics of its chemical constituents, pharmacological effects, pharmacokinetic and toxicity were descriptively summarized. A total of 9706 studies were identified and 83 of them were included. As a result, 134 chemical constituents were identified, including 26 alkaloids, 29 compounds found in essential oils, 13 diterpenoids, two carbohydrates, two sterols, 18 amino acids, six nucleosides, four nucleobases, four fatty acids, three lignans, and 27 elements. Thirteen pharmacological effects of FTB were identified, including anti-cancer, tracheobronchial relaxation, antitussive, expectorant, anti-muscarinic, anti-inflammation, anti-thyroid, regulation of blood rheology, antiulcer, anti-diarrhea, pain suppression, antioxidation and neuroprotection. These pharmacological activities may be mainly attributed to the alkaloids in FTB. Further phytochemical, pharmacological and network pharmacological studies are recommended.
\end{abstract}

Keywords: natural product; traditional medicine; complementary and alternative medicine; herbal medicine; review

\section{Introduction}

Fritillariae Thunbergii Bulbus (FTB) is also known as Zhe bei mu or Xiang bei mu in Chinese, Setsubaimo in Japanese and Jeolpaemo in Korean [1]. Fritillariae Thunbergii belongs to the Fritillariae (Liliaceae) family and FTB has been widely used as an antitussive herb for thousands of years in China [2]. Fritillariae Thunbergii is widely cultivated in the south-eastern coastal, south-central and eastern areas of China, such as Zhejiang, Jiangsu, Anhui and Hunan provinces [2]. FTB is a significant traditional Chinese herb with bitter and cold properties, entering the Lung and Heart channels [2]. It is generally prescribed as one of the ingredients of herbal formula, such as Danggui Beimu Kushen Wan (Chinese Angelica, Fritillaria and Flavescent Sophora Pill; DBKW) in traditional and contemporary clinical practice [3,4]. Recently, investigating potential treatments from ancient records has been employed as a strategy to direct novel drug discovery [5]. Modern studies that are recorded in the Zhong Yao Da Ci Dian (Great Compendium of Chinese Medicines) [6] and Zhong 
Hua Ben Cao (Chinese Pharmacopoeia) [7] indicated that the pharmacological effects of FTB include antitussive, tracheobronchial relaxation, anti-muscarinic, expectorant and pain suppression with a wide range of chemical constituents, such as peimine and peiminine. With the development of modern methodological approaches, an increasing number of new chemical constituents (e.g., frithunbol A and frithunbol B) [8], and pharmacological effects (e.g., anti-cancer activity [9] and neuroprotection [8]) have been found in FTB. Therefore, there is a need to thoroughly review FTB from traditional and modern perspectives to assist further drug discovery. This study aimed to review traditional uses, phytochemical, pharmacodynamic, pharmacokinetic and toxicity characteristics of FTB and provide comprehensive and evidence-based information to practitioners and researchers.

\section{Traditional Uses of FTB}

The Zhong Hua Yi Dian (Encyclopedia of Traditional Chinese Medicine; ZHYD) CD-ROM, containing 1156 ancient books, was searched to identify potential classic literature of FTB [10]. FTB was first documented in the book Ben Cao Hui Yan (BCHY, Discourse on Herbal Medicine, NI Zhumo, 1624, Ming Dynasty), with its actions described as breaking blood and detoxication. In addition, five classic books, including Ben Cao Zheng Yao (Evidence of Materia Medica, LI Zhongzi, 1673, Qing dynasty), Ben Jing Feng Yuan (Doctrine of Origin, ZHANG Lu, 1695, Qing dynasty), Ben Cao Gang Mu Shi Yi (Supplement to the Compendium of Materia Medica, ZHAO Xuemin, 1765, Qing dynasty), Ben Cao Bian Du (Simple Materia Medica, ZHANG Bingcheng, 1887, Qing dynasty) and Ben Cao Zheng Yi (Merits of Herbal Medicine, ZHANG Shanlei, 1920, Republic of China), specified that the unique actions of FTB were to clear the Lung and calm the Liver, disperse the Lung-Qi to relieve depression, clear the Heart and reduce the heat, which were not found in other Fritillariae species, such as Fritillariae Cirrhosae Bulbus (Chuan bei $\mathrm{mu}$ ) and Bulbus Bolbostemmatis Rhizoma ( $\mathrm{Tu}$ Bei $\mathrm{Mu}$ ). In summary, FTB acts to clear heat, resolve phlegm, relieve cough, remove toxicity and disperse abscesses and nodules. A number of herbal formulas containing FTB were identified. Supplementary file 1 presents some example formulas containing FTB as an ingredient in ZHYD. Herbal ingredients in Supplementary file 1 are listed as Chinese pinyin name as per the nomenclature list of commonly used Chinese herbal medicines published by the Chinese Medicine Board of Australia [11]. Today, some of them are continuously used by practitioners in evidence-based Chinese medicine clinical practice, such as DBKW, Guang Bi Shu Zhan Tang (Throat Tuberculosis Decoction), Xiao Lei Wan (Eliminate Scrofula Pill), Xing Su Yin (Apricot Kernel and Perilla Leaf Decoction), Qing Jin Ning Sou Tang (Clear the Lung and Stop Cough Decoction), Tuo Li Pai Nong Tang (Support the Interior and Drain Pus Decoction), Ren Shen Bai Du San (Ginseng Powder to Overcome Toxin) and Xian Fang Huo Ming Yin (Sublime Formula for Sustaining Life).

In terms of the indications of FTB, Ben Cao Zheng (Orthodox Materia Medica, ZHANG Jiebin, 1624, Ming dynasty) recorded that it could be used for a number of conditions, including lung abscess, lung atrophy, cough, dyspnea, hematemesis, epistaxis, hematochezia, hematuria, jaundice, difficult urination, pharyngitis, hemorrhoid, anal fistula, acute mastitis, scrofula and any furuncles, carbuncle, abscess and other inflammation and swelling. Ben Cao Cong Xin (New Revised Materia Medica, WU Yiluo, 1751, Qing dynasty) claimed that FTB could be utilized for patients with flu induced by wind-phlegm. Ben Cao Gang Mu Shi Yi provided further descriptions that the indications of FTB could be extended to any respiratory disorders which are due to wind-fire and phlegm. As shown in Supplementary file 1, those formulas had been widely used in various disciplines along with the development of Chinese medicine, including otorhinolaryngology, ophthalmology, external medicine, gynecology, obstetrics, galactophore and infectious diseases.

\section{Modern Exploration of FTB}

The following seven English and four Chinese electronic databases were searched from their respective inceptions to 2 November 2018: PubMed, EMBASE, CINAHL, Science Direct, Scopus, ProQuest, Allied and Complementary Medicine Database, CNKI, CQVIP, Wanfangdata and CBM. 
Keywords used to identify potential studies included: Zhe bei mu and its English, Latin, Japanese, Korean, botanical and pharmaceutical names and their synonyms. The reference list of review articles was screened manually for potential studies.

Original experimental studies on phytochemistry and pharmacology, or the studies containing phytochemical or pharmacological tests, published in English or Chinese, were considered for inclusion, regardless of the type of experimental studies (e.g., in vivo and in vitro). The studies were included if they investigated a single raw herb with species and specific medicinal parts of FTB listed in the Pharmacopoeia of the People's Republic of China. The literature was excluded if they are review articles or do not include original phytochemical or pharmacological studies, or did not use a raw herb with appropriate species or medicinal parts of FTB, or directly used chemical compounds instead of a raw herb as the research material, or published in a non-English or non-Chinese language.

One reviewer (HL) screened the searched literature and extracted the following data to a predesigned Excel form: Compound name, molecular formula, molecular weight, molecular structure and analysis methods for phytochemical studies; and compound name, study type, characteristics of sample, interventions, duration, outcome measures, main results for pharmacological studies. Corresponding molecular structures were further searched in the PubChem database to obtain the PubChem CID/SID number. The molecular structures that could not be found in PubChem were drawn manually using the software ChemDraw. All included studies were divided into two groups: Studies reporting a voucher number (SRV) and studies not reporting voucher number (SNRV). A voucher specimen number is one of the most significant requirements for phytological studies, since it ensures the reliability of botanical taxon and the repeatability of published studies [12,13]. The data was checked and confirmed by the second reviewer (ML). When any discrepancies between the two reviewers occurred, they were discussed with the third party (AY) to resolve the issues. A descriptive summary was performed to obtain the characteristics of the extracted data.

A total of 9706 experimental studies were identified adhering to the search strategies mentioned above. Eighty-three studies meeting the inclusion criteria were included for analysis, including 63 phytochemical studies $[8,14-75]$ and 30 pharmacological studies $[8,9,17,27,33,45,49,56,59,73,75-94]$, as 10 studies reported both phytochemical and pharmacological effects of FTB $[8,17,27,33,45,49,56,59,73,75]$. Figure 1 details the selection process of included studies. 

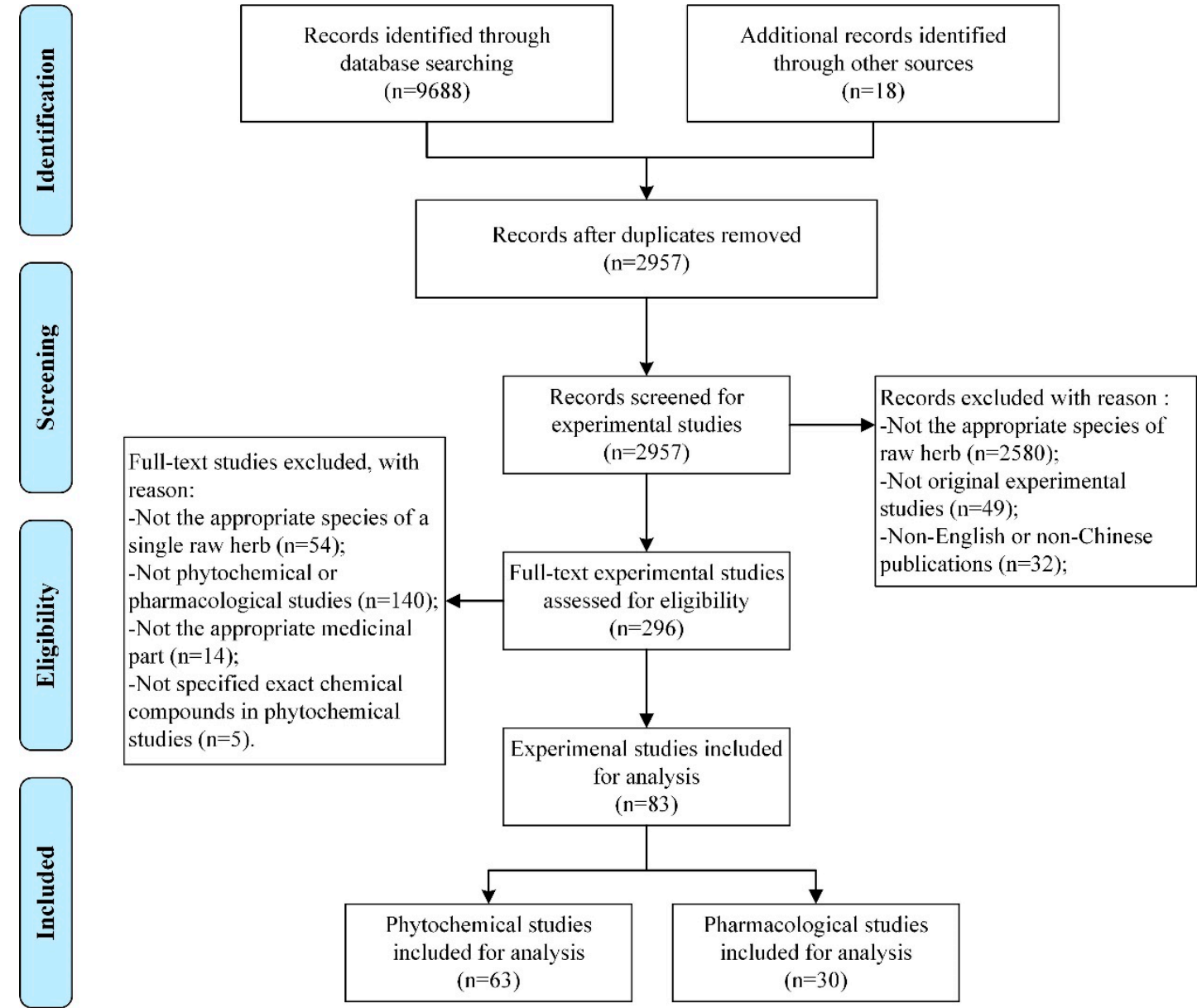

Figure 1. Flowchart of the selection process of Fritillariae Thunbergii Bulbus studies.

\subsection{Phytochemistry of FTB}

A total of 134 chemical constituents were identified, including 26 alkaloids (A1-A26), 29 compounds found in essential oils (B1-B29), 13 diterpenoids (C1-C13), two carbohydrates (D1-D2), two sterols (E1-E2), 18 amino acids (F1-F18), six nucleosides (G1-G6), four nucleobases (H1-H4), four fatty acids (I1-I4), three lignans (J1-J3), and 27 elements (K1-K27). Table 1 details the characteristics of chemical constituents isolated from FTB. 
Table 1. Summary of the 134 chemical constituents isolated from Fritillariae Thunbergii Bulbus.

\begin{tabular}{|c|c|c|c|c|c|c|}
\hline \multirow{2}{*}{ No. } & \multirow{2}{*}{$\begin{array}{l}\text { Derivatives and } \\
\text { Constitutions }\end{array}$} & \multirow{2}{*}{$\begin{array}{l}\text { Molecular } \\
\text { Formula }\end{array}$} & \multirow{2}{*}{$\begin{array}{l}\text { PubChem } \\
\text { CID/SID }\end{array}$} & \multirow{2}{*}{$\begin{array}{c}\text { Molecule } \\
\text { Weight }(\mathrm{g} / \mathrm{mol})\end{array}$} & \multicolumn{2}{|r|}{ Method } \\
\hline & & & & & SRV Group & SNRV Group \\
\hline A & Alkaloids $(n=26)$ & & & & & \\
\hline A1 & Peimine & $\mathrm{C}_{27} \mathrm{H}_{45} \mathrm{NO}_{3}$ & 131900 & 431.661 & $\begin{array}{c}\text { HPLC [33]; HPLC-ELSD [44,60,63]; HPLC-ESI/MS [70]; } \\
\text { HPLC-MS [21]; UPLC-ELSD [26]; } \\
\text { LC/ESI-QTOF-MS/MS [72]; Pre-column derivatization } \\
\text { HPLC [38]; GC [55]; }{ }^{1} \text { HNMR } /{ }^{13} \text { CNMR/HR-FAB-MS [8] }\end{array}$ & $\begin{array}{c}\text { HPLC [18,20]; HPLC-ELSD [17,27-29,32,36,47,51]; } \\
\text { HSCCC-ELSD [31]; UPLC-CAD/HPLC-ELSD [42]; } \\
\text { UHPLC-MS/MS [59]; LC-LTQ-Orbitrap MS [22]; } \\
\text { LC-TRAP-MS/LC-ESI-MS [45]; ESI-MS [73]; TLCS [23,48,57]; } \\
\text { 13CNMR [69]; Determination of colorimetry [23]; } \\
\text { RRLC-MS/MS [49]; MS/IR/1NNMR/ } /{ }^{13} \text { CNMR [75]; GC [37]; } \\
\text { GC direct [17]; Indirect UV detection [41]; Paper partition } \\
\text { chromatography [46]; Aid-dye ofnrimetry [24,39] }\end{array}$ \\
\hline $\mathrm{A} 2$ & Peiminine & $\mathrm{C}_{27} \mathrm{H}_{43} \mathrm{NO}_{3}$ & 167691 & 429.645 & 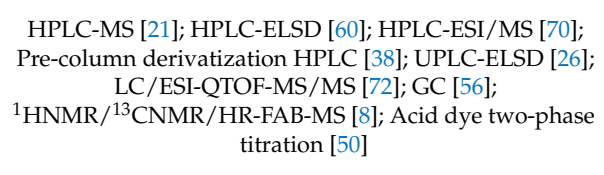 & $\begin{array}{l}\text { HPLC [17,18,20]; HPLC-ELSD [27-29,32,36,47]; } \\
\text { HPLC-CAD/HPLC-ELSD [42]; HSCCC-ELSD [31]; ESI-MS [73]; } \\
\text { UHPLC-MS/MS [59]; TLCS [23,48,57]; LC-LTQ-Orbitrap MS }[22 \text {; } \\
\text { LC-TRAP-MS/LC-ESI-MS [45]; }{ }^{13} \text { CNMR [69]; Determination of } \\
\text { colorimetry [23]; RRLC-MS/MS [49]; MS/IR/ } /{ }^{1} \text { HNMR/ } \\
{ }^{13} \text { CNMR [75]; GC [37]; GC direct [17]; Indirect UV detection [41] }\end{array}$ \\
\hline A3 & Zhebeinine & $\mathrm{C}_{27} \mathrm{H}_{45} \mathrm{NO}_{3}$ & 21121503 & 431.661 & $\mathrm{~N} / \mathrm{A}$ & TLC [69]; ${ }^{13}$ CNMR [69] \\
\hline A4 & Zhebeinone & $\mathrm{C}_{27} \mathrm{H}_{43} \mathrm{NO}_{3}$ & NF (Figure 2) & 429.645 & N/A & GC-MS [65] \\
\hline A5 & Ebeiedine & $\mathrm{C}_{27} \mathrm{H}_{45} \mathrm{NO}_{2}$ & 101324888 & 415.662 & $\begin{array}{l}\text { HPLC [33]; GC [56]; LC/ESI-QTOF-MS/MS [72]; } \\
{ }^{1} \text { HNMR } /{ }^{13} \text { CNMR/HR-FAB-MS [8] }\end{array}$ & GC [37]; GC direct [17]; LC-TRAP-MS/LC-ESI-MS [45] \\
\hline A6 & Ebeiedinone & $\mathrm{C}_{27} \mathrm{H}_{43} \mathrm{NO}_{2}$ & 102062796 & 413.646 & GC [56]; ${ }^{1} \mathrm{HNMR} /{ }^{13} \mathrm{CNMR} / \mathrm{HR}-\mathrm{FAB}-\mathrm{MS}[8]$ & $\begin{array}{l}\text { GC [37]; GC direct [17]; LC-TRAP-MS/LC-ESI-MS [44]; } \\
\text { LC-LTQ-Orbitrap MS }{ }^{n} \text { [22]; HPLC-ELSD [17,36] }\end{array}$ \\
\hline A7 & Isoverticine & $\mathrm{C}_{27} \mathrm{H}_{45} \mathrm{NO}_{3}$ & 21573744 & 431.661 & $\begin{array}{l}\text { GC [56]; LC/ESI-QTOF-MS/MS [72]; } \\
{ }^{1} \text { HNMR } /{ }^{13} \text { CNMR/HR-FAB-MS [8] }\end{array}$ & $\begin{array}{l}\text { GC [37]; GC direct [17]; LC-TRAP-MS/LC-ESI-MS [45]; HPLC-ELSD } \\
\text { [17,36]; ESI-MS [73]; TLCS [57] }\end{array}$ \\
\hline A8 & Suchengbeisine & $\mathrm{C}_{27} \mathrm{H}_{43} \mathrm{NO}_{3}$ & 102112537 & 429.645 & HPLC [33]; ${ }^{1} \mathrm{HNMR} /{ }^{13} \mathrm{CNMR} / \mathrm{HR}-\mathrm{FAB}-\mathrm{MS}$ [8] & N/A \\
\hline A9 & Peimisine & $\mathrm{C}_{27} \mathrm{H}_{41} \mathrm{NO}_{3}$ & 161294 & 427.629 & LC/ESI-QTOF-MS/MS [72]; UPLC-ELSD [26] & $\begin{array}{l}\text { LC-TRAP-MS/LC-ESI-MS [45]; HPLC-ELSD [17,36,47]; TLC [68]; } \\
\text { TLCS [571: LC-LTO-Orbitrap MSn [22]: RRLC-MS/MS [49] }\end{array}$ \\
\hline A10 & Peimisine-N-oxide & $\mathrm{C}_{27} \mathrm{H}_{42} \mathrm{NO}_{4}$ & $\mathrm{NF}$ & 444.636 & $\mathrm{~N} / \mathrm{A}$ & LC-LTQ-Orbitrap MS ${ }^{n}$ [22] \\
\hline A11 & Zhebeininoside & $\mathrm{C}_{33} \mathrm{H}_{55} \mathrm{NO}_{8}$ & NF (Figure 2) & 593.802 & LC/ESI-QTOF-MS/MS [72] & $\begin{array}{l}\text { LC-TRAP-MS/LC-ESI-MS [45]; LC-LTQ-Orbitrap MS }{ }^{\mathrm{n}} \text { [22]; } \\
{ }_{13} \text { CNMR/Infrared spectra analysis [67]; ESI-MS [73] }\end{array}$ \\
\hline $\begin{array}{l}\text { A12 } \\
\text { A13 }\end{array}$ & $\begin{array}{l}\text { Verticinone-3- } \beta \text {-D-glucoside } \\
\text { Puqietinone }\end{array}$ & $\begin{array}{l}\mathrm{C}_{33} \mathrm{H}_{53} \mathrm{NO}_{8} \\
\mathrm{C}_{2} \mathrm{H}_{47} \mathrm{NO}_{2}\end{array}$ & $\begin{array}{l}90479257 \\
10693900\end{array}$ & $\begin{array}{l}591.786 \\
429.689\end{array}$ & $\begin{array}{l}\text { LC/ESI-QTOF-MS/MS [72] } \\
\text { LC/ESI-OTOF-MS/MS [72] }\end{array}$ & $\begin{array}{ll}\text { N/A } & -1 / 2 \\
\text { N/A }\end{array}$ \\
\hline A14 & Puqiedinone & $\mathrm{C}_{27} \mathrm{H}_{43} \mathrm{NO}_{2}$ & 126149 & 413.646 & LC/ESI-QTOF-MS/MS [72] & $\begin{array}{l}\text { N/A } \\
\text { LC-TRAP-MS/LC-ESI-MS [45]; LC-LTQ-Orbitrap MS [22] }\end{array}$ \\
\hline A15 & Puqiedine & $\mathrm{C}_{27} \mathrm{H}_{45} \mathrm{NO}_{2}$ & 101400593 & 415.662 & LC/ESI-QTOF-MS/MS [72] & $\begin{array}{l}\text { LC-TRAP-MS/LC-ESI-MS [45]; ESI-MS [73] } \\
\text { [1-1 }\end{array}$ \\
\hline A16 & Eduardine & $\mathrm{C}_{27} \mathrm{H}_{43} \mathrm{NO}_{2}$ & NF (Figure 2) & 413.646 & N/A & GC-MS [66] \\
\hline A17 & Zhebeirine & $\mathrm{C}_{27} \mathrm{H}_{43} \mathrm{NO}_{2}$ & NF (Figure 2) & 413.646 & N/A & GC-MS [66]; ESI-MS [73] \\
\hline A18 & 2,3-deoxyladenosine & $\mathrm{C}_{10} \mathrm{H}_{11} \mathrm{~N}_{5} \mathrm{O}_{2}$ & NF & 233.231 & N/A & ESI-MS [73] \\
\hline A19 & $\mathrm{N}$-demethylpuqietinone & $\mathrm{C}_{27} \mathrm{H}_{45} \mathrm{NO}_{2}$ & 11304576 & 415.662 & $\mathrm{~N} / \mathrm{A}$ & ESI-MS [73] \\
\hline A20 & Puqienine B & $\mathrm{C}_{28} \mathrm{H}_{45} \mathrm{NO}_{2}$ & 11419389 & 443.672 & $\mathrm{~N} / \mathrm{A}$ & ESI-MS [73] \\
\hline A21 & Zhebeinone-3- $\beta$-D-glucoside & $\mathrm{C}_{33} \mathrm{H}_{55} \mathrm{NO}_{8}$ & NF (Figure 2) & 593.802 & N/A & ESI-MS [73] \\
\hline A22 & Peiminoside & $\mathrm{C}_{33} \mathrm{H}_{55} \mathrm{NO}_{7}$ & 90479565 & 577.803 & $\mathrm{~N} / \mathrm{A}$ & Paper partition chromatography [46] \\
\hline A23 & Frithunbol A & $\mathrm{C}_{27} \mathrm{H}_{41} \mathrm{NO}_{4}$ & NF (Figure 2) & 443.628 & ${ }^{1} \mathrm{HNMR} /{ }^{13} \mathrm{CNMR} / \mathrm{HR}-\mathrm{FAB}-\mathrm{MS}$ [8] & N/A \\
\hline A24 & Frithunbol B & $\mathrm{C}_{27} \mathrm{H}_{42} \mathrm{NO}_{3}$ & NF (Figure 2) & 428.637 & ${ }^{1} \mathrm{HNMR} /{ }^{13} \mathrm{CNMR} / \mathrm{HR}-\mathrm{FAB}-\mathrm{MS}[8]$ & N/A \\
\hline A25 & Eduardinine & $\mathrm{C}_{27} \mathrm{H}_{45} \mathrm{NO}_{2}$ & NF (Figure 2) & 415.662 & ${ }^{1} \mathrm{HNMR} /{ }^{13} \mathrm{CNMR} / \mathrm{HR}-\mathrm{FAB}-\mathrm{MS}[8]$ & $\mathrm{N} / \mathrm{A}$ \\
\hline A26 & 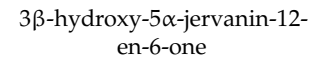 & $\mathrm{C}_{27} \mathrm{H}_{42} \mathrm{NO}_{3}$ & NF (Figure 2) & 428.637 & ${ }^{1} \mathrm{HNMR} /{ }^{13} \mathrm{CNMR} / \mathrm{HR}$-FAB-MS [8] & N/A \\
\hline
\end{tabular}


Table 1. Cont

\begin{tabular}{|c|c|c|c|c|c|c|}
\hline \multirow{2}{*}{ No. } & \multirow{2}{*}{$\begin{array}{l}\text { Derivatives and } \\
\text { Constitutions }\end{array}$} & \multirow{2}{*}{$\begin{array}{l}\text { Molecular } \\
\text { Formula }\end{array}$} & \multirow{2}{*}{$\begin{array}{l}\text { PubChem } \\
\text { CID/SID }\end{array}$} & \multirow{2}{*}{$\begin{array}{c}\text { Molecule } \\
\text { Weight }(\mathrm{g} / \mathrm{mol})\end{array}$} & \multicolumn{2}{|c|}{ Method } \\
\hline & & & & & SRV Group & SNRV Group \\
\hline B & $\begin{array}{c}\text { Compounds from essential } \\
\text { oils }(n=29)\end{array}$ & & & & & \\
\hline B1 & d-elemene & $\mathrm{C}_{15} \mathrm{H}_{24}$ & 12309449 & 204.357 & $\mathrm{~N} / \mathrm{A}$ & GC-MS [16] \\
\hline B2 & $\delta$-selinene & $\mathrm{C}_{15} \mathrm{H}_{24}$ & 520383 & 204.357 & N/A & GC-MS [16] \\
\hline B3 & Tetradecanoic acid & $\mathrm{C}_{14} \mathrm{H}_{28} \mathrm{O}_{2}$ & 11005 & 228.376 & N/A & GC-MS [16] \\
\hline B4 & Pentadecanoic acid & $\mathrm{C}_{15} \mathrm{H}_{30} \mathrm{O}_{2}$ & 13849 & 242.403 & $\mathrm{~N} / \mathrm{A}$ & GC-MS [16] \\
\hline B5 & $\begin{array}{l}\text { Hexadecanoic acid, methyl } \\
\text { ester }\end{array}$ & $\mathrm{C}_{17} \mathrm{H}_{34} \mathrm{O}_{2}$ & 8181 & 270.457 & $\mathrm{~N} / \mathrm{A}$ & GC-MS $[16,25]$ \\
\hline B6 & 9-hexadecenoic acid & $\mathrm{C}_{16} \mathrm{H}_{30} \mathrm{O}_{2}$ & 5282745 & 254.414 & $\mathrm{~N} / \mathrm{A}$ & GC-MS [16] \\
\hline B7 & N-hexadecanoic acid & $\mathrm{C}_{16} \mathrm{H}_{32} \mathrm{O}_{2}$ & 985 & 256.43 & N/A & GC-MS [16] \\
\hline B8 & Kaur-15-ene & $\mathrm{C}_{20} \mathrm{H}_{32}$ & 521318 & 272.476 & $\mathrm{~N} / \mathrm{A}$ & GC-MS [16] \\
\hline B9 & Heptadecanoic acid & $\mathrm{C}_{17} \mathrm{H}_{34} \mathrm{O}_{2}$ & 10465 & 270.457 & N/A & GC-MS [16] \\
\hline B10 & Kaurene & $\mathrm{C}_{20} \mathrm{H}_{32}$ & 91746569 & 272.476 & $\mathrm{~N} / \mathrm{A}$ & GC-MS [16] \\
\hline B11 & $\begin{array}{c}\text { 9,12-Octadecadienoic acid } \\
\text { (Z,Z)-, } \\
\text { methyl ester }\end{array}$ & $\mathrm{C}_{19} \mathrm{H}_{34} \mathrm{O}_{2}$ & 5284421 & 294.479 & $\mathrm{~N} / \mathrm{A}$ & GC-MS [16] \\
\hline B12 & 9-Tetradecenal, (Z)- & $\mathrm{C}_{14} \mathrm{H}_{26} \mathrm{O}$ & 5364471 & 210.361 & N/A & GC-MS [16] \\
\hline B13 & $\begin{array}{l}\text { 9,12-Octadecadienoic acid, } \\
\text { methyl ester, (E, E)- }\end{array}$ & $\mathrm{C}_{19} \mathrm{H}_{34} \mathrm{O}_{2}$ & 135058711 & 294.479 & $\mathrm{~N} / \mathrm{A}$ & GC-MS [16] \\
\hline B14 & Oleic acid & $\mathrm{C}_{18} \mathrm{H}_{34} \mathrm{O}_{2}$ & 445639 & 282.468 & N/A & GC-MS [16] \\
\hline B15 & Linoleic acid, ethyl ester & $\mathrm{C}_{2036} \mathrm{O}_{2}$ & 5282184 & 308.506 & $\mathrm{~N} / \mathrm{A}$ & GC-MS [16] \\
\hline B16 & Butylated hydroxytoluene & $\mathrm{C}_{15} \mathrm{H}_{24} \mathrm{O}$ & 31404 & 220.356 & $\mathrm{~N} / \mathrm{A}$ & GC-MS (Du et al., 2018) \\
\hline B17 & $\begin{array}{l}\text { L-(+)-Ascorbic acid 2,6- } \\
\text { dihexadecanoate }\end{array}$ & $\mathrm{C}_{38} \mathrm{H}_{68} \mathrm{O}_{8}$ & 54722209 & 652.954 & N/A & GC-MS (Du et al., 2018) \\
\hline B18 & Ethyl 9-hexadecenoate & $\mathrm{C}_{18} \mathrm{H}_{34} \mathrm{O}_{2}$ & 5364759 & 282.468 & N/A & GC-MS [25] \\
\hline B19 & $\begin{array}{l}\text { Hexadecanoic acid, ethyl ester } \\
1 \mathrm{H}-\mathrm{Naphtho}[2,1-\mathrm{B}] \text { pyran, }\end{array}$ & $\mathrm{C}_{18} \mathrm{H}_{36} \mathrm{O}_{2}$ & 12366 & 284.484 & N/A & GC-MS [25] \\
\hline B20 & $\begin{array}{c}\text { 3-ethenyldodecahydro-3,4a, } 7,7, \\
\text { 10a-pentamethyl- }\end{array}$ & $\mathrm{C}_{20} \mathrm{H}_{34} \mathrm{O}$ & 273540178 & 290.491 & N/A & GC-MS [25] \\
\hline B21 & Kaur-16-ene & $\mathrm{C}_{20} \mathrm{H}_{32} \mathrm{O}$ & 520687 & 272.476 & N/A & GC-MS [25] \\
\hline B22 & $\begin{array}{l}\text { 9,11-Octadecadienoic acid, } \\
\text { methyl ester, (E, E)- }\end{array}$ & $\mathrm{C}_{19} \mathrm{H}_{34} \mathrm{O}_{2}$ & 319301067 & 294.479 & $\mathrm{~N} / \mathrm{A}$ & GC-MS [25] \\
\hline B23 & 9,12-Octadecadienoic acid & $\mathrm{C}_{18} \mathrm{H}_{32} \mathrm{O}_{2}$ & 5282457 & 280.452 & N/A & GC-MS [25] \\
\hline B24 & Linoleic acid, ethyl ester & $\mathrm{C}_{20} \mathrm{H}_{36} \mathrm{O}_{2}$ & 5282184 & 308.506 & $\mathrm{~N} / \mathrm{A}$ & GC-MS [25] \\
\hline B25 & $\begin{array}{l}\text { Octadecanoic acid, ethyl ester } \\
2(1 \mathrm{H}) \text {-Phenanthrenone, }\end{array}$ & $\mathrm{C}_{20} \mathrm{H}_{40} \mathrm{O}_{2}$ & 8122 & 312.538 & $\mathrm{~N} / \mathrm{A}$ & GC-MS [25] \\
\hline B26 & 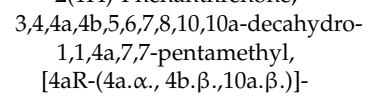 & $\mathrm{C}_{19} \mathrm{H}_{30} \mathrm{O}$ & 621255 & 274.448 & N/A & GC-MS [25] \\
\hline B27 & 3-Methyleneandrostan-17-ol & $\mathrm{C}_{20} \mathrm{H}_{32} \mathrm{O}$ & 625647 & 288.475 & N/A & GC-MS [25] \\
\hline B28 & $\begin{array}{l}\text { Androst-4-en-3-one, } \\
\text { 17-hydroxy-, (17.ß.) }\end{array}$ & $\mathrm{C}_{19} \mathrm{H}_{28} \mathrm{O}_{2}$ & 50049744 & 288.431 & $\mathrm{~N} / \mathrm{A}$ & GC-MS [25] \\
\hline B29 & $\begin{array}{l}\text { Podocarp-7-en-3.ß.-ol, } \\
\text { 13.ß.-methyl-13-vinyl- }\end{array}$ & $\mathrm{C}_{20} \mathrm{H}_{32} \mathrm{O}$ & 620519 & 288.475 & N/A & GC-MS [25] \\
\hline
\end{tabular}


Table 1. Cont

\begin{tabular}{|c|c|c|c|c|c|c|}
\hline \multirow{2}{*}{ No. } & \multirow{2}{*}{$\begin{array}{l}\text { Derivatives and } \\
\text { Constitutions }\end{array}$} & \multirow{2}{*}{$\begin{array}{l}\text { Molecular } \\
\text { Formula }\end{array}$} & \multirow{2}{*}{$\begin{array}{l}\text { PubChem } \\
\text { CID/SID }\end{array}$} & \multirow{2}{*}{$\begin{array}{c}\text { Molecule } \\
\text { Weight (g/mol) }\end{array}$} & \multicolumn{2}{|c|}{ Method } \\
\hline & & & & & SRV Group & SNRV Group \\
\hline $\mathrm{C}$ & Diterpenoids $(n=13)$ & & & & & \\
\hline $\mathrm{C} 1$ & Isopimaran-19-ol & $\mathrm{C}_{20} \mathrm{H}_{32} \mathrm{O}$ & 75399514 & 288.475 & N/A & ${ }^{1} \mathrm{HNMR} /{ }^{13} \mathrm{CNMR}$ [35] \\
\hline $\mathrm{C} 2$ & $\begin{array}{l}\text { Isopimaran-19-oic acid, methyl } \\
\text { ester }\end{array}$ & $\mathrm{C}_{21} \mathrm{H}_{32} \mathrm{O}_{2}$ & NF (Figure 2) & 316.485 & $\mathrm{~N} / \mathrm{A}$ & ${ }^{1} \mathrm{HNMR} /{ }^{13} \mathrm{CNMR}[34,35]$ \\
\hline C3 & Ent-kauran-16 $\beta, 17-$ diol & $\mathrm{C}_{20} \mathrm{H}_{34} \mathrm{O}_{2}$ & NF (Figure 2) & 306.49 & $\mathrm{~N} / \mathrm{A}$ & TLC [68]; ${ }^{1} \mathrm{HNMR} /{ }^{13} \mathrm{CNMR}[34,35]$ \\
\hline $\mathrm{C} 4$ & Ent-kauran-16 $\alpha$, 17-diol & $\mathrm{C}_{20} \mathrm{H}_{34} \mathrm{O}_{2}$ & NF (Figure 2) & 306.49 & N/A & ${ }^{1} \mathrm{HNMR} /{ }^{13} \mathrm{CNMR}[34,35]$ \\
\hline C5 & Ent-16 $\beta, 17$-epoxy-kaurane & $\mathrm{C}_{20} \mathrm{H}_{32} \mathrm{O}$ & 79592848 & 288.475 & N/A & ${ }^{1} \mathrm{HNMR} /{ }^{13} \mathrm{CNMR}[35]$ \\
\hline C6 & Ent-16 $\alpha$-methoxy-kauran-17-ol & $\mathrm{C}_{21} \mathrm{H}_{36} \mathrm{O}_{2}$ & 51842049 & 320.517 & N/A & ${ }^{1} \mathrm{HNMR} /{ }^{13} \mathrm{CNMR}[35]$ \\
\hline $\mathrm{C} 7$ & Ent-kaur-15-en-17-ol & $\mathrm{C}_{20} \mathrm{H}_{32} \mathrm{O}$ & 3082069 & 288.475 & N/A & ${ }^{1} \mathrm{HNMR} /{ }^{13} \mathrm{CNMR}[35]$ \\
\hline C8 & Trans-communol & $\mathrm{C}_{20} \mathrm{H}_{32} \mathrm{O}$ & 51909318 & 288.475 & $\mathrm{~N} / \mathrm{A}$ & ${ }^{1} \mathrm{HNMR} /{ }^{13} \mathrm{CNMR}[34]$ \\
\hline C9 & $\begin{array}{l}\text { Trans-comminic acid, methyl } \\
\text { ester }\end{array}$ & $\mathrm{C}_{21} \mathrm{H}_{32} \mathrm{O}_{2}$ & NF (Figure 2) & 316.485 & $\mathrm{~N} / \mathrm{A}$ & ${ }^{1} \mathrm{HNMR} /{ }^{13} \mathrm{CNMR}$ [34] \\
\hline $\mathrm{C} 10$ & Ent-17-norkauran-16-one & $\mathrm{C}_{19} \mathrm{H}_{30} \mathrm{O}$ & 12740861 & 274.448 & $\mathrm{~N} / \mathrm{A}$ & ${ }^{1} \mathrm{HNMR} /{ }^{13} \mathrm{CNMR}$ [34] \\
\hline $\mathrm{C} 11$ & Ent-15ß,16-epoxy-kauran-17-ol & $\mathrm{C}_{20} \mathrm{H}_{32} \mathrm{O}_{2}$ & 51511087 & 304.474 & N/A & ${ }^{1} \mathrm{HNMR} /{ }^{13} \mathrm{CNMR}[34]$ \\
\hline $\mathrm{C} 12$ & $\begin{array}{l}\text { Ent-16ß-hydroxy-kauran-17-yl } \\
\text { ent-kaur-15-en-17-oate }\end{array}$ & $\mathrm{C}_{40} \mathrm{H}_{63} \mathrm{O}_{3}$ & NF (Figure 2) & 591.941 & $\mathrm{~N} / \mathrm{A}$ & ${ }^{1} \mathrm{HNMR} /{ }^{13} \mathrm{CNMR}[34]$ \\
\hline $\mathrm{C} 13$ & Ent-(16S)-atisan-13, 17-oxide & $\mathrm{C}_{20} \mathrm{H}_{32} \mathrm{O}$ & 50418337 & 288.475 & $\mathrm{~N} / \mathrm{A}$ & ${ }^{1} \mathrm{HNMR} /{ }^{13} \mathrm{CNMR}$ [34] \\
\hline D & Carbohydrates $(n=2)$ & & & & & \\
\hline D1 & $\beta$-D-glucose4-1 $\beta$-D-galactose & $\mathrm{C}_{12} \mathrm{H}_{22} \mathrm{O}_{11}$ & $\mathrm{NF}$ & 342.297 & N/A & HPLC-ELSD [19] \\
\hline D2 & Sucrose & $\mathrm{C}_{12} \mathrm{H}_{22} \mathrm{O}_{11}$ & 5988 & 342.297 & N/A & HPLC-ELSD [19] \\
\hline $\mathrm{E}$ & Sterols $(n=2)$ & & & & & \\
\hline E1 & $\beta$-sitosterol & $\mathrm{C}_{29} \mathrm{H}_{50} \mathrm{O}$ & 222284 & 414.718 & N/A & $\operatorname{TLC}[68]$ \\
\hline E2 & Daucosterol & $\mathrm{C}_{35} \mathrm{H}_{60} \mathrm{O}_{6}$ & 5742590 & 576.859 & N/A & TLC [68] \\
\hline F & Amino acids $(n=18)$ & & & & & \\
\hline F1 & Glycine & $\mathrm{C}_{2} \mathrm{H}_{5} \mathrm{NO}_{2}$ & 750 & 75.067 & $\mathrm{~N} / \mathrm{A}$ & HPLC [64] \\
\hline F2 & Leucine & $\mathrm{C}_{6} \mathrm{H}_{13} \mathrm{NO}_{2}$ & 6106 & 131.175 & N/A & HPLC [64] \\
\hline F3 & Methionine & $\mathrm{C}_{5} \mathrm{H}_{11} \mathrm{NO}_{2} \mathrm{~S}$ & 6137 & 149.208 & N/A & HPLC [64] \\
\hline F4 & Tyrosine & $\mathrm{C}_{9} \mathrm{H}_{11} \mathrm{NO}_{3}$ & 6057 & 181.191 & N/A & HPLC [64] \\
\hline F5 & Histidine & $\mathrm{C}_{6} \mathrm{H}_{9} \mathrm{~N}_{3} \mathrm{O}_{2}$ & 6274 & 155.157 & N/A & HPLC [64] \\
\hline F6 & Threonine & $\mathrm{C}_{4} \mathrm{H}_{9} \mathrm{NO}_{3}$ & 6288 & 119.12 & N/A & HPLC [64] \\
\hline F7 & Alanine & $\mathrm{C}_{3} \mathrm{H}_{7} \mathrm{NO}_{2}$ & 5950 & 89.094 & $\mathrm{~N} / \mathrm{A}$ & HPLC [64] \\
\hline F8 & Isoleucine & $\mathrm{C}_{6} \mathrm{H}_{13} \mathrm{NO}_{2}$ & 6306 & 131.175 & N/A & HPLC [64] \\
\hline F9 & Tryptophan & $\mathrm{C}_{11} \mathrm{H}_{12} \mathrm{~N}_{2} \mathrm{O}_{2}$ & 6305 & 204.229 & N/A & HPLC [64] \\
\hline F10 & Cystine & $\mathrm{C}_{6} \mathrm{H}_{12} \mathrm{~N}_{2} \mathrm{O}_{4} \mathrm{~S}_{2}$ & 67678 & 240.292 & N/A & HPLC [64] \\
\hline F11 & Lysine & $\mathrm{C}_{6} \mathrm{H}_{14} \mathrm{~N}_{2} \mathrm{O}_{2}$ & 5962 & 146.19 & N/A & HPLC [64] \\
\hline F12 & Aspartic acid & $\mathrm{C}_{4} \mathrm{H}_{7} \mathrm{NO}_{4}$ & 5960 & 133.103 & N/A & HPLC [64] \\
\hline F13 & Valine & $\mathrm{C}_{5} \mathrm{H}_{11} \mathrm{NO}_{2}$ & 6287 & 117.148 & N/A & HPLC [64] \\
\hline F14 & Phenylalanine & $\mathrm{C}_{8} \mathrm{H}_{8} \mathrm{O}_{2}$ & 6140 & 165.192 & N/A & HPLC [64] \\
\hline F15 & Proline & $\mathrm{C}_{5} \mathrm{H}_{9} \mathrm{NO}_{2}$ & 145742 & 115.132 & N/A & HPLC [64] \\
\hline F16 & Serine & $\mathrm{C}_{3} \mathrm{H}_{7} \mathrm{NO}_{3}$ & 5951 & 105.093 & N/A & HPLC [64] \\
\hline F17 & Glutamic acid & $\mathrm{C}_{5} \mathrm{H}_{9} \mathrm{NO}_{4}$ & 33032 & 147.13 & N/A & HPLC [64] \\
\hline F18 & Arginine & $\mathrm{C}_{6} \mathrm{H}_{14} \mathrm{~N}_{4} \mathrm{O}_{2}$ & 6322 & 174.204 & N/A & HPLC [64] \\
\hline
\end{tabular}


Table 1. Cont.

\begin{tabular}{|c|c|c|c|c|c|c|}
\hline \multirow{2}{*}{ No. } & \multirow{2}{*}{$\begin{array}{l}\text { Derivatives and } \\
\text { Constitutions }\end{array}$} & \multirow{2}{*}{$\begin{array}{l}\text { Molecular } \\
\text { Formula }\end{array}$} & \multirow{2}{*}{$\begin{array}{l}\text { PubChem } \\
\text { CID/SID }\end{array}$} & \multirow{2}{*}{$\begin{array}{c}\text { Molecule } \\
\text { Weight }(\mathrm{g} / \mathrm{mol})\end{array}$} & \multicolumn{2}{|c|}{ Method } \\
\hline & & & & & SRV Group & SNRV Group \\
\hline G & Nucleosides $(n=6)$ & & & & \multirow{3}{*}{ 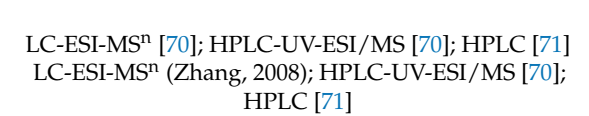 } & \\
\hline G1 & Uridine & $\mathrm{C}_{9} \mathrm{H}_{12} \mathrm{~N}_{2} \mathrm{O}_{6}$ & 6029 & 244.203 & & HPLC [64] \\
\hline G2 & Guanosine & $\mathrm{C}_{10} \mathrm{H}_{13} \mathrm{~N}_{5} \mathrm{O}_{5}$ & 6802 & 283.244 & & HPLC [64] \\
\hline G3 & Adenosine & $\mathrm{C}_{10} \mathrm{H}_{13} \mathrm{~N}_{5} \mathrm{O}_{4}$ & 60961 & 267.245 & 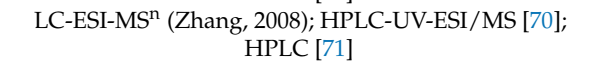 & HPLC [64] \\
\hline G4 & Thymidine & $\mathrm{C}_{10} \mathrm{H}_{14} \mathrm{~N}_{2} \mathrm{O}_{5}$ & 5789 & 242.231 & LC-ESI-MS ${ }^{n}$ [70]; HPLC-UV-ESI/MS [70] & HPLC [64] \\
\hline G5 & Cytidine & $\mathrm{C}_{9} \mathrm{H}_{13} \mathrm{~N}_{3} \mathrm{O}_{5}$ & 6175 & 243.219 & N/A & HPLC [64] \\
\hline G6 & Inosine & $\mathrm{C}_{10} \mathrm{H}_{12} \mathrm{~N}_{4} \mathrm{O}_{5}$ & 6021 & 268.229 & $\mathrm{~N} / \mathrm{A}$ & HPLC [64] \\
\hline $\mathrm{H}$ & Nucleobases $(n=4)$ & & & & & \\
\hline H1 & Adenine & $\mathrm{C}_{5} \mathrm{H}_{5} \mathrm{~N}_{5}$ & 190 & 135.13 & $\begin{array}{l}\text { LC-ESI-MS }^{n} \text { (Zhang, 2008); HPLC-UV-ESI/MS (Zhang, } \\
\text { 2008) }\end{array}$ & HPLC [64] \\
\hline $\mathrm{H} 2$ & Hypoxanthine & $\mathrm{C}_{5} \mathrm{H}_{4} \mathrm{~N}_{4} \mathrm{O}$ & 790 & 136.114 & N/A & HPLC [64] \\
\hline H3 & Uracil & $\mathrm{C}_{4} \mathrm{H}_{4} \mathrm{~N}_{2} \mathrm{O}_{2}$ & 1174 & 112.088 & N/A & HPLC [64] \\
\hline $\mathrm{H} 4$ & Thymine & $\mathrm{C}_{5} \mathrm{H}_{6} \mathrm{~N}_{2} \mathrm{O}_{2}$ & 1135 & 126.115 & N/A & HPLC [64] \\
\hline I & \multirow{5}{*}{$\begin{array}{c}\text { Fatty acids }(n=4) \\
\text { Vernolic acid } \\
\text { 2-monopalmitin } \\
\text { 13(R)-hydroxy-octadeca-(9Z,11E } \\
\text {,15Z)-trien-oic acid } \\
\text { Picropodophyllotoxin }\end{array}$} & & & & & \\
\hline I1 & & $\mathrm{C}_{18} \mathrm{H}_{32} \mathrm{O}_{3}$ & 6449780 & 296.451 & $\mathrm{~N} / \mathrm{A}$ & ESI-MS [73] \\
\hline I2 & & $\mathrm{C}_{19} \mathrm{H}_{38} \mathrm{O}_{4}$ & 123409 & 330.509 & N/A & ESI-MS [73] \\
\hline I3 & & $\mathrm{C}_{18} \mathrm{H}_{30} \mathrm{O}_{3}$ & 643726 & 294.435 & $\mathrm{~N} / \mathrm{A}$ & ESI-MS [73] \\
\hline I4 & & $\mathrm{C}_{22} \mathrm{H}_{22} \mathrm{O}_{8}$ & 72435 & 414.41 & N/A & ${ }^{13} \mathrm{CNMR} /$ Infrared spectra analysis [67] \\
\hline $\mathrm{J}$ & Lignans $(n=3)$ & & & & & \\
\hline $\mathrm{J} 1$ & Octahydrocurcumin & $\mathrm{C}_{21} \mathrm{H}_{28} \mathrm{O}_{6}$ & 11068834 & 376.449 & N/A & ESI-MS [73] \\
\hline $\mathrm{J} 2$ & Zhebeiresinol & $\mathrm{C}_{14} \mathrm{H}_{16} \mathrm{O}_{6}$ & 192547 & 280.276 & N/A & ESI-MS [73]; ${ }^{1} \mathrm{HNMR} /{ }^{13} \mathrm{CNMR}$ [30] \\
\hline $\mathrm{J} 3$ & Sauriol B & $\mathrm{C}_{21} \mathrm{H}_{28} \mathrm{O}_{6}$ & 15965508 & 376.449 & N/A & ESI-MS [73] \\
\hline $\mathrm{K}$ & Elements $(n=27)$ & & & & & \\
\hline K1 & Aluminum & $\mathrm{Al}$ & 5359268 & 26.982 & ICP-OES [62] & FAAS [61]; GFAAS [61] \\
\hline K2 & Arsenic & As & 5359596 & 74.922 & ICP-AES [43]; ICP-OES [14] & ICP-AES [74]; ICP-OES [15]; ICAP [55]; FAAS [61]; GFAAS [61] \\
\hline K3 & Boron & B & 5462311 & 10.81 & ICP-OES $[14,62]$ & N/A \\
\hline K4 & Barium & $\mathrm{Ba}$ & 5355457 & 137.327 & ICP-AES [58]; ICP-OES [14] & N/A \\
\hline K5 & Bismuth & $\mathrm{Bi}$ & 5359367 & 208.98 & ICP-OES [14] & $\mathrm{N} / \mathrm{A}$ \\
\hline K6 & Calcium & $\mathrm{Ca}$ & 5460341 & 40.078 & ICP-OES [62] & FAAS $[52,53,61]$; GFAAS $[61]$ \\
\hline K7 & Cadmium & $\mathrm{Cd}$ & 23973 & 112.414 & ICP-AES [43]; ICP-OES [14,62] & $\begin{array}{l}\text { ICP-AES [74]; ICP-OES [15]; ICAP [55]; FAAS [61]; GFAAS [61] } \\
\text { AAS [40] }\end{array}$ \\
\hline K8 & Cobalt & Co & 104730 & 58.933 & ICP-AES [58]; ICP-OES [14] & FAAS [61]; GFAAS [61] \\
\hline K9 & Chromium & $\mathrm{Cr}$ & 23976 & 51.996 & ICP-AES [58] & FAAS [61]; GFAAS [61]; AAS [40] \\
\hline K10 & Copper & $\mathrm{Cu}$ & 23978 & 63.546 & ICP-AES [43,58]; ICP-OES [14] & $\begin{array}{l}\text { ICP-AES [74]; ICP-OES [15]; ICAP [55]; FAAS [52,53,61]; } \\
\text { GFAAS [61]: AAS [40] }\end{array}$ \\
\hline K11 & Iron & $\mathrm{Fe}$ & 23925 & 55.845 & ICP-AES [43,58]; ICP-OES [14] & ICP-AES [74]; FAAS [52,53,61]; GFAAS [61]; AAS [40] \\
\hline K12 & Mercury & $\mathrm{Hg}$ & 23931 & 200.592 & ICP-AES [43]; ICP-OES [14] & ICP-OES [15] \\
\hline K13 & Indium & In & 5359967 & 114.818 & ICP-OES [14] & N/A \\
\hline K14 & Potassium & $\mathrm{K}$ & 5462222 & 39.098 & ICP-OES [62] & FAAS [53,61]; GFAAS [61] \\
\hline
\end{tabular}


Table 1. Cont.

\begin{tabular}{|c|c|c|c|c|c|c|}
\hline \multirow{2}{*}{ No. } & \multirow{2}{*}{$\begin{array}{l}\text { Derivatives and } \\
\text { Constitutions }\end{array}$} & \multirow{2}{*}{$\begin{array}{l}\text { Molecular } \\
\text { Formula }\end{array}$} & \multirow{2}{*}{$\begin{array}{l}\text { PubChem } \\
\text { CID/SID }\end{array}$} & \multirow{2}{*}{$\begin{array}{c}\text { Molecule } \\
\text { Weight (g/mol) }\end{array}$} & \multicolumn{2}{|c|}{ Method } \\
\hline & & & & & SRV Group & SNRV Group \\
\hline $\mathbf{K}$ & Elements $(n=27)$ & & & & & \\
\hline K15 & Lithium & $\mathrm{Li}$ & 3028194 & 6.94 & ICP-AES [43] & N/A \\
\hline K16 & Magnesium & $\mathrm{Mg}$ & 5462224 & 24.305 & ICP-AES [43]; ICP-OES [62] & FAAS [53,61]; GFAAS [61]; AAS [40] \\
\hline K17 & Manganese & $\mathrm{Mn}$ & 23930 & 54.938 & ICP-AES [43,58]; ICP-OES [14] & ICP-AES [74]; FAAS [53,61]; GFAAS [61]; ICAP [55] \\
\hline K18 & Molybdenum & Mo & 23932 & 95.95 & ICP-OES [62] & N/A \\
\hline K19 & Sodium & $\mathrm{Na}$ & 5360545 & 22.99 & ICP-OES [62] & FAAS [53,61]; GFAAS [61] \\
\hline K20 & Nickel & $\mathrm{Ni}$ & 935 & 58.693 & ICP-AES [58]; ICP-OES $[14,62]$ & ICAP [55] \\
\hline $\mathrm{K} 21$ & Phosphorus & $\mathrm{P}$ & 5462309 & 30.974 & ICP-OES [62] & N/A \\
\hline K22 & Lead & $\mathrm{Pb}$ & 5352425 & 207.2 & ICP-AES [43]; ICP-OES [14] & ICP-OES [15]; AAS [40]; ICAP [55] \\
\hline K23 & Sulfur & $\mathrm{S}$ & 5362487 & 32.06 & ICP-AES [43] & N/A \\
\hline K24 & Selenium & Se & 6326970 & 78.971 & N/A & Spectrophotometry [54] \\
\hline K25 & Strontium & $\mathrm{Sr}$ & 5359327 & 87.62 & ICP-AES [43,58]; ICP-OES & FAAS [61]; GFAAS [61] \\
\hline K26 & Vanadium & $\mathrm{V}$ & 23990 & 50.941 & ICP-AES [58] & N/A \\
\hline К27 & Zinc & $\mathrm{Zn}$ & 23994 & 65.379 & ICP-AES [43,58]; ICP-OES [14] & $\begin{array}{l}\text { ICP-AES [74]; ICP-OES; ICAP [55]; FAAS [52,53,61]; GFAAS [61]; } \\
\text { AAS [40] }\end{array}$ \\
\hline
\end{tabular}

Note: N/A: Not applicable; NF: Not found; SRV: Studies reporting a voucher number; SNRV: Studies not reporting voucher number; Corresponding molecular structures refer to PubChem and Figure 2. 


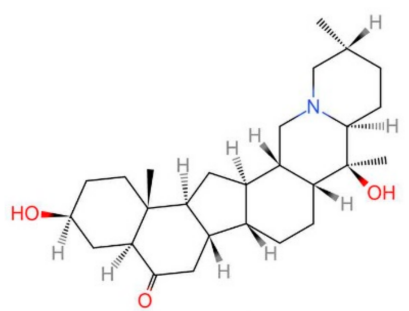

A4

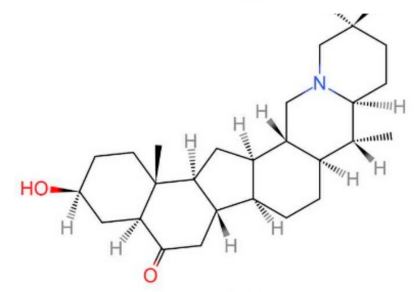

A17<smiles>CC1=C2C[C@]3(C)[C@@H](CC(=O)[C@@H]4CC(O)CC[C@@]43C)[C@@H]2CC[C@@]12O[C@H]1C[C@H](C)CN[C@H]1[C@H]2C</smiles>

A24<smiles>C=C[C@]1(C)CC[C@H]2C(=CC[C@]3(O)[C@@](C)(C(C)=O)CCC[C@@]23C)C1</smiles>

C2

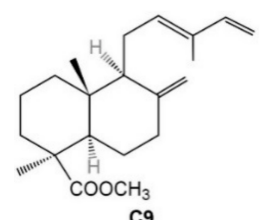

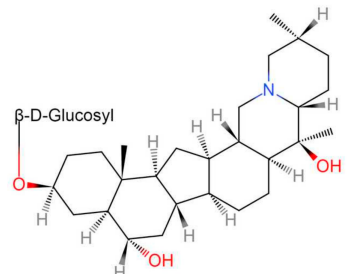

A11

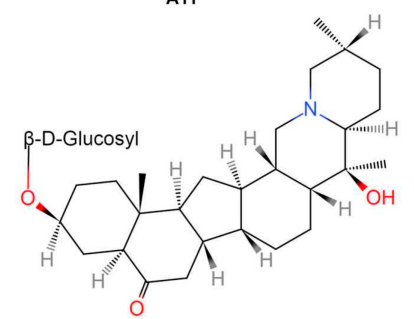

A21

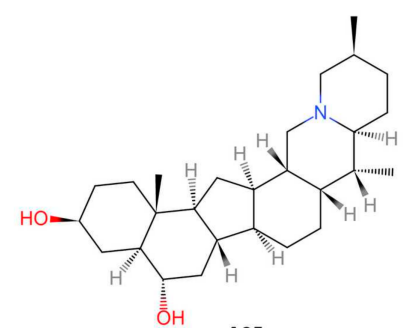

A25

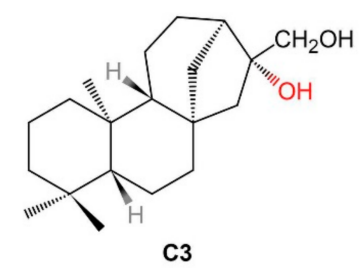

C3

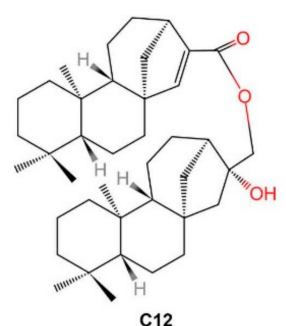

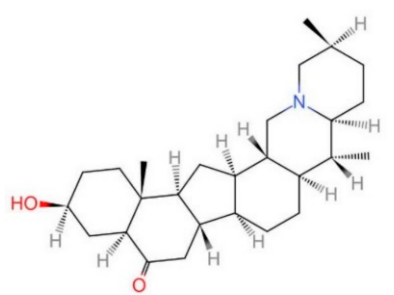

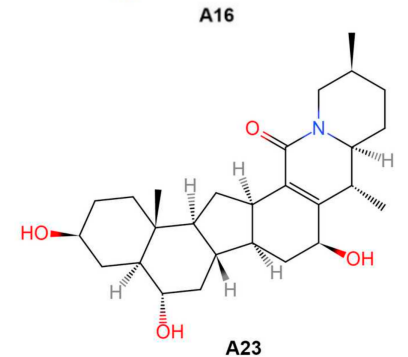

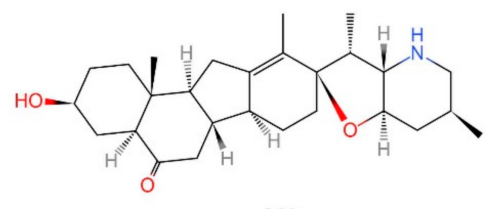

A26<smiles>CC1(C)CCC[C@@]2(C)[C@H]3CC[C@]4(O)[C@@H](CO)[C@]4(CC[C@@H]12)C3</smiles>

Figure 2. Molecular structures of the 14 chemical constituents of Fritillariae Thunbergii Bulbus. Note: A4: Zhebeinone; A11: Zhebeininoside; A16: Eduardine; A17: Zhebeirine; A21: Zhebeinone-3- $\beta$-D- glucoside; A23: Frithunbol A; A24: Frithunbol B; A25: Eduardinine; A26: 3 $\beta$-hydroxy-5 $\alpha$-jervanin-12-en-6-one; C2: Isopimaran-19-oic acid, methyl ester; C3: Ent-kauran-16 $\beta$, 17-diol; C4: Ent-kauran-16 $\alpha$, 17-diol; C9: Trans-comminic acid, methyl ester; C12: Ent-16ß-hydroxy-kauran-17-yl ent-kaur-15-en-17-oate.

Within 63 included phytochemical studies, there are 17 SRV (47 constituents isolated) $[8,14,21$, $26,33,38,43,44,50,56,58,60,62,63,70-72]$ and 46 SNRV (118 constituents isolated) [15-20,22-32,34-37, 39-42,45-49,51-55,57,59,61,64-66,69,73-75]. Thirty-one chemical constituents were identified in both SRV and SNRV groups, including nine alkaloids (A1, A2, A5, A6, A7, A9, A11, A14 and A15), four nucleosides (G1-G4), one nucleobases (I1), and 17 elements (K1, K2, K6-K12, K14, K16, K17, K19, K20, $\mathrm{K} 22, \mathrm{~K} 25$ and K27). In terms of the molecular structures, 117 chemical constituents were found in the PubChem database that refers to the corresponding PubChem CID/SID, however, 17 constituents were not available in PubChem. Amount of the 17 chemical components, 14 of the molecular structures (A4, 
A11, A16, A17, A27, A30-A33, C2-C4, C9 and C12) could be found in the included studies and three of them (A10, A22 and D1) could not be found anywhere. Details of the 14 molecular structures were presented in Figure 2.

\subsubsection{Alkaloids}

Current studies mainly focused on the alkaloids which are the fundamental components of FTB [34]. Among 26 isolated alkaloids in FTB, $0.080 \%$ of peimine (A1) and peiminine (A2) used for identification and quality control as listed in the Pharmacopeia of People's Republic of China [2]. These two compounds were first isolated in 1960 using the paper partition chromatography method [46] and then they were consistently found by over 40 research teams using more than 20 different methods from 1960 to 2018 [8,17,18,20-24,26-29,31-33,36-39,41,42,44,45,47-49,51,56,57,59,60,63,70,72,73,75]. The in vivo and in vitro experimental studies identified the biological activities of peimine (A1) and peiminine (A2), such as anti-cancer activity [49], tracheobronchial relaxation [17], anti-muscarinic activity [75], anti-inflammatory activity [33,88], and pain suppression [88], which indicates they may have great potential in clinical practice. In addition, some isomers were also reported, including peimine (A1) [33], zhebeinine (A3) [68] and isoverticine (A7) [56] with the same molecular formula $\mathrm{C}_{27} \mathrm{H}_{45} \mathrm{NO}_{3}$, peiminine (A2) [21], zhebeinone (A4) [65] and suchengbeisine (A8) [33] with the same molecular formula $\mathrm{C}_{27} \mathrm{H}_{43} \mathrm{NO}_{3}$, ebeiedine (A5) [33], puqiedine (A15) [72], N-demethylpuqietinon (A19) [73] and eduardinine (A25) [8] with the same molecular formula $\mathrm{C}_{27} \mathrm{H}_{45} \mathrm{NO}_{2}$, ebeiedinone (A6) [56], puqiedinone (A14) [72], eduardine (A16) [66] and zhebeirine (A17) [66] with the same molecular formula $\mathrm{C}_{27} \mathrm{H}_{43} \mathrm{NO}_{2}$, and frithunbol B (A24) [8] and 3 $\beta$-hydroxy-5 $\alpha$-jervanin-12-en-6-one (A26) [8] with the same molecular formula $\mathrm{C}_{27} \mathrm{H}_{42} \mathrm{NO}_{3}$. Another two new steroidal alkaloids were isolated and identified in 2018 [8]. They are the colorless gum called frithunbol $A$ (A23) and frithunbol B (A24) with the structures $5 \alpha$-cevanin-13-ene-3 $\beta, 6 \alpha, 16 \beta$-triol-18-one and $3 \beta$-hydroxy- $5 \alpha$-jerv-12-en-6-one respectively, presented in Figure 2. Frithunbol B (A24) could reduce nitric oxide $(\mathrm{NO})$ in vitro and it may possess neuroprotective activity [8].

\subsubsection{Compounds from Essential Oils}

A total of 29 compounds found in essential oils in FTB were investigated in two recent experimental studies using the gas chromatography-mass spectrometry method. One study reported 15 of them, including $\delta$-elemene (B1), $\delta$-selinene (B2), tetradecanoic acid (B3), pentadecanoic acid (B4), hexadecanoic acid, methyl ester (B5), 9-hexadecenoic acid (B6), n-hexadecanoic acid (B7), kaur-15-ene (B8), heptadecanoic acid (B9), kaurene (B10), 9,12-octadecadienoic acid (Z,Z)-, methyl ester (B11), 9-tetradecenal, (Z)- (B12), 9,12-octadecadienoic acid, methyl ester, (E, E)- (B13), oleic acid (B14), and linoleic acid, ethyl ester (B15) [16]. Among these 15 constituents, n-hexadecanoic acid (B7), 9,12-octadecadienoic acid, methyl ester, (E, E)- (B13), and oleic acid (B14) were the three primary compounds found in essential oils in FTB accounting for $53.46 \%, 26.96 \%$ and $9.34 \%$ respectively. Another study also found hexadecanoic acid, methyl ester (B5), and reported another 14 chemical compounds, including butylated hydroxytoluene (B16), L-(+)-Ascorbic acid 2,6-dihexadecanoate (B17), ethyl 9-hexadecenoate (B18), hexadecanoic acid, ethyl ester (B19), 1H-naphtho [2,1-B] pyran, 3-ethenyldodecahydro-3,4a,7,7,10a-pentamethyl- (B20), kaur-16-ene (B21), 9,11-octadecadienoic acid, methyl ester, (E, E)- (B22), 9,12-octadecadienoic acid (B23), linoleic acid, ethyl ester (B24), octadecanoic acid, ethyl ester (B25), 2(1H)-phenanthrenone, 3,4,4a,4b,5,6,7,8,10,10a-decahydro-1,1,4a,7,7-pentamethyl,

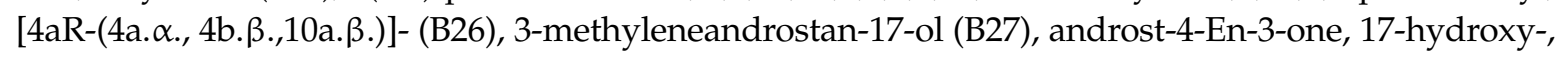

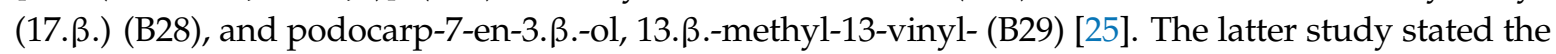
proportion of four main compounds found in essential oils: Linoleic acid, ethyl ester (B24; 36.93\%), kaur-16-ene (B21; 22.85\%), hexadecanoic acid, methyl ester (B5; 10.67\%), and 3-methyleneandrostan-17-ol (B27; 10.59\%). However, the proportion of hexadecanoic acid, methyl ester (B5) in the former study was only $0.6 \%$ [25]. It is worth noting that different compounds from essential oils with different proportions from FTB have been identified, due to the different isolation methods. The former study soaked FTB 
powders for $12 \mathrm{~h}$ and distilled it for $10 \mathrm{~h} \mathrm{[16]} \mathrm{whereas} \mathrm{the} \mathrm{soak} \mathrm{time} \mathrm{and} \mathrm{distillation} \mathrm{time} \mathrm{in} \mathrm{the} \mathrm{later}$ study were one hour and five hours respectively [25]. Further studies should consider utilizing the appropriate isolation method to achieve selective isolation of target compounds. However, at the current stage, there is no solid evidence to identify which isolation method is better.

\subsubsection{Diterpenoids}

Two studies conducted by the same Japanese research team in 1984 focused on the diterpenoids in FTB $[34,35]$. Thirteen diterpenoids were identified using the nuclear magnetic resonance methods (1HNMR and 13CNMR), including isopimaran-19-ol (C1), isopimaran-19-oic acid, methyl ester (C2), ent-kauran-16 $\beta$, 17-diol (C3), ent-kauran-16 $\alpha$, 17-diol (C4), ent-16 $\beta$, 17-epoxy-kaurane (C5), ent-16 $\alpha$-methoxy-kauran-17-ol (C6), ent-kaur-15-en-17-ol (C7), trans-communol (C8), trans-comminic acid, methyl ester (C9), ent-17-norkauran-16-one (C10), ent-15 $\beta, 16$-epoxy-kauran-17-ol (C11), ent-16ß-hydroxy-kauran-17-yl ent-kaur-15-en-17-oate (C12), and ent-(16S)-atisan-13, 17-oxide (C13) [34,35]. However, no further studies, after 1984, were found on the investigation of the diterpenoids or the specific diterpenoids identified from FTB. As diterpenoids in other Chinese herbs may perform diverse biological activities, such as anti-cancer activity [95], anti-inflammatory activity [96] and antioxidative activity [97], further studies which focus attention on diterpenoids in FTB may be important.

\subsubsection{Nucleosides}

Three studies identified six nucleosides from FTB using three different methods (LC-ESI-MSn [70], HPLC-UV-ESI/MS [70] and HPLC [64,71]), including uridine (G1), guanosine (G2), adenosine (G3), thymidine (G4), cytidine (G5), and inosine (G6). One of the studies highlighted that the content of nucleosides in FTB could be used as an index for the identification and quality control of FTB [64]. However, to date, none of the included studies evaluated the pharmacological activities of nucleosides isolated from FTB. As the basic building-block of DNA and RNA, nucleosides from other plants possess many biological activities, such as anti-cancer activity [98], antibacterial activity [99], and antiviral activity [100]. It is worth investigating nucleosides in FTB for novel drug discovery.

\subsubsection{Elements}

A total of 27 elements were found for identification and quality control purposes $[14,15,40,43$, $52,53,55,58,61,62,74]$. Eight of them are trace elements that may have vital biological activities in the human body, including cobalt, copper, iron, manganese, molybdenum, sulfur, selenium and zinc [14, $15,40,43,52,54,55,58,61,62,74]$. Another six trace elements without any known biological activities were identified in FTB, including arsenic, cadmium, chromium, mercury, nickel and lead [14,15,40,43,55,58, $61,62,74]$. They have toxic activities that may damage the lipids, proteins, enzymes and DNA even in low dose [101]. All the toxic elements in the included studies were under the maximum limited level listed in the Pharmacopeia of the People's Republic of China $[14,15,40,43,52,53,55,58,61,62,74]$.

\subsubsection{Other Constituents}

Apart from above chemical components, 33 chemical compounds belonging to six categories were isolated and identified, which are used for identification, as well as quality control, including two carbihydrates ( $\beta$-D-glucose4-1 $\beta$-D-galactose (D1) and sucrose (D2)) [19], two sterols ( $\beta$-sitosterol (E1) and daucosterol (E2)) [68], 18 amino acids (glycine (F1), leucine (F2), methionine (F3), tyrosine (F4), histidine (F5), threonine (F6), alanine (F7), isoleucine (F8), tryptophan (F9), cystine (F10), lysine (F11), aspartic acid (F12), valine (F13), phenylalanine (F14), proline (F15), serine (F16), glutamic acid (F17) and arginine (F18)) [64], four nucleobases (adenine (H1), hypoxanthine (H2), uracil (H3) and thymine (H4)) [64,70], four fatty acids (vernolic acid (I1), 2-monopalmitin (I2), 13(R)-hydroxy-octadeca-(9Z,11E,15Z)-trien-oic acid (I3) and picropodophyllotoxin (I4)) [67,73], and three lgnans (octahydrocurcumin (J1), zhebeiresinol (J2) and sauriol B (J3)) [30,73]. 


\subsection{Pharmacology of FTB}

There are 23 studies focused on the pharmacological effects of FTB, including anti-cancer, tracheobronchial relaxation, antitussive, expectorant, anti-muscarinic, anti-inflammation, anti-thyroid, regulation of blood rheology, antiulcer, anti-diarrhea, pain suppression, antioxidation and neuroprotection (Figure 3). Characteristics of the 23 included pharmacological effects are summarized in Table 2 


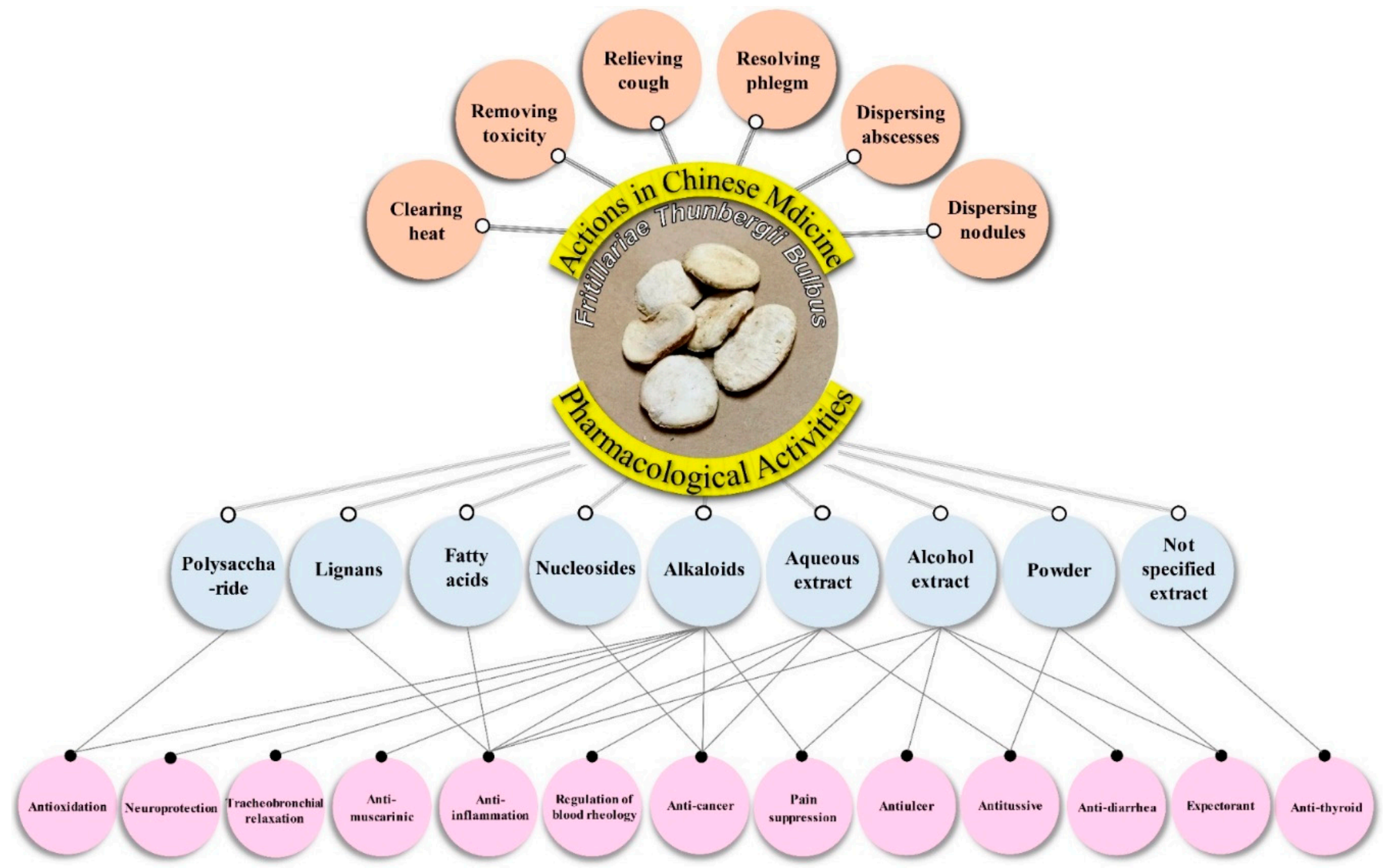

Figure 3. Multiple pharmacological effects of Fritillariae Thunbergii Bulbus. 
Table 2. Characteristics of the 23 included studies relevant to the mechanisms of actions of Fritillariae Thunbergii Bulbus.

\begin{tabular}{|c|c|c|c|c|c|c|}
\hline $\begin{array}{l}\text { Pharmacological } \\
\text { Effects/ } \\
\text { Included Studies }\end{array}$ & Study Type & Extract & $\begin{array}{l}\text { Characteristics of the } \\
\text { Sample }\end{array}$ & Interventions & Duration & Primary Results \\
\hline \multicolumn{7}{|l|}{ 1. Anti-cancer } \\
\hline \multirow[t]{2}{*}{ Li et al., 2013 [80] } & In vitro & Total alkaloids & $\begin{array}{l}\text { Human lung } \\
\text { adenocarcinoma } \\
\text { parental cells A549; } \\
\text { Resistant cells } \\
\text { A549/DDP }\end{array}$ & $\begin{array}{c}\text { Cytotoxicity: FTB 12.5, 25, 50, 100, } 200 \\
\text { mg/L and vehicle } 0.5 \% \text { CMC-Na. } \\
\text { Multidrug resistance reversal effect: FTB } \\
9 \text { mg/L, cyclosporine A } 1 \mathrm{mg} / \mathrm{L} \text { or } \\
\text { tetrandrine } 1 \mathrm{mg} / \mathrm{L} \text { plus DDP (final } \\
\text { concentration: } 0.01,0.1,1,10,100 \mathrm{mg} / \mathrm{L}) \\
\text { MDR1 mRNA and P-gp expression: } \\
\text { A549/DDP + vehicle; A549/DDP + FTB } \\
\text { 9 mg/L; A549/DDP + DDP } 14 \mathrm{mg} / \mathrm{L} ; \\
\text { A549/DDP + DDP } 14 \mathrm{mg} / \mathrm{L}+\mathrm{FTB} \\
9 \mathrm{mg} / \mathrm{L} ; \text { A549 + vehicle }\end{array}$ & $72 \mathrm{~h}$ & $\begin{array}{l}\mathrm{IC}_{50} \text { of TAF to A549: } 141 \pm 5 \mathrm{mg} / \mathrm{L} ; \\
\mathrm{IC}_{50} \text { of TAF to A549/DDP: } 298 \pm 22 \mathrm{mg} / \mathrm{L} \text {; } \\
\text { FTB was superior to closporine A and } \\
\text { tetrandrines in increasing the reversal fold; } \\
\text { FTB alone was superior to vehicle in } \\
\text { decreasing the MDR1 mRNA and P-gp } \\
\text { expression. }\end{array}$ \\
\hline & In vivo & Total alkaloids & $\begin{array}{l}60 \mathrm{BALB} / \mathrm{c} \text { nude mice } \\
\text { (A549/DDP model) }\end{array}$ & $\begin{array}{c}\text { Vehicle 0.5\% CMC-Na; DDP } 5 \text { mg/kg, } \\
\text { ig, qd; TAF } 2 \text { mg/kg, ig, qd; DDP } \\
5 \mathrm{mg} / \mathrm{kg} \text { + FTB } 0.5 \mathrm{mg} / \mathrm{kg} \text {, ig, qd; DDP } \\
5 \mathrm{mg} / \mathrm{kg} \text { + FTB } 1 \mathrm{mg} / \mathrm{kg} \text {, ig, qd; DDP } \\
5 \mathrm{mg} / \mathrm{kg} \text { + FTB } 2 \mathrm{mg} / \mathrm{kg} \text {, ig, qd }\end{array}$ & 13 days & $\begin{array}{l}\mathrm{DDP}+\mathrm{TAF} \text { was superior to DDP alone in } \\
\text { increasing the tumor inhibitory rate. }\end{array}$ \\
\hline Liu et al., 2015 [9] & In vitro & $\begin{array}{l}\text { Total alkaloids; } \\
\text { Total } \\
\text { nucleosides }\end{array}$ & $\begin{array}{c}\text { Human } \\
\text { hepatocellular } \\
\text { carcinoma cell line } \\
\text { HepG2, Resistant cell } \\
\text { line HepG2/MDR }\end{array}$ & $\begin{array}{l}\text { Blank control; P-gp positive inhibitor } \\
\text { verapamil; Total alkaloid, nucleoside, or } \\
\text { polysaccharide } 5 \mu \mathrm{g} / \mathrm{mL} \text { respectively; } \\
\text { Total alkaloid, nucleoside, or } \\
\text { polysaccharide } 50 \mu \mathrm{g} / \mathrm{mL} \text { respectively }\end{array}$ & $1 \mathrm{~h}$ & $\begin{array}{l}\text { Total alkaloids or Total nucleosides in FTB } \\
\text { was superior to the control in increasing the } \\
\text { restriction of efflux activity of P-gp. }\end{array}$ \\
\hline \multirow[t]{2}{*}{ Yang et al., 2005 [91] } & In vitro & $\begin{array}{l}\text { Aqueous } \\
\text { extract }\end{array}$ & $\begin{array}{c}\text { Human lung } \\
\text { adenocarcinoma } \\
\text { parental cells } \mathrm{LM}_{2}\end{array}$ & $\begin{array}{c}\text { Blank control; FZ + FTB }(75+75,25+25, \\
5+5,1+1 \mathrm{mg} / \mathrm{mL} \text { respectively); FZ }(75 \\
25,5 \mathrm{mg} / \mathrm{mL} \text { respectively); FTB }(75,25 \\
5 \mathrm{mg} / \mathrm{mL} \text { respectively) }\end{array}$ & $48 \mathrm{~h}$ & $\begin{array}{l}\text { FTB alone was superior to control and FZ + } \\
\text { FTB in increasing the apoptosis rate. }\end{array}$ \\
\hline & In vivo & $\begin{array}{l}\text { Aqueous } \\
\text { extract }\end{array}$ & $\begin{array}{l}142 \mathrm{SPF} \mathrm{C}_{57} \text { mice } \\
\text { (Human lung } \\
\text { adenocarcinoma } \\
\text { parental cells } \mathrm{LM}_{2} \\
\text { model) }\end{array}$ & $\begin{array}{c}\text { Blank control; FZ + FTB }(0.8+1.6 \mathrm{~g} / \mathrm{kg} \\
0.64+1.28 \mathrm{~g} / \mathrm{kg}, 0.51+1.02 \mathrm{~g} / \mathrm{kg} \\
\text { respectively, ig, qd); FZ }(0.8 \mathrm{~g} / \mathrm{kg}, \\
0.64 \mathrm{~g} / \mathrm{kg}, 0.51 \mathrm{~g} / \mathrm{kg} \text { respectively, ig, qd); } \\
\text { FTB }(1.6 \mathrm{~g} / \mathrm{kg}, 1.28 \mathrm{~g} / \mathrm{kg}, 1.02 \mathrm{~g} / \mathrm{kg} \\
\text { respectively, ig, qd) }\end{array}$ & 18 days & $\begin{array}{l}\text { FTB alone was superior to control and FZ }+ \\
\text { FTB in increasing the tumor inhibitory rate } \\
\text { and reducing the number of metastases. }\end{array}$ \\
\hline
\end{tabular}


Table 2. Cont.

\begin{tabular}{|c|c|c|c|c|c|c|}
\hline $\begin{array}{l}\text { Pharmacological } \\
\text { Effects/ } \\
\text { Included Studies }\end{array}$ & Study Type & Extract & $\begin{array}{l}\text { Characteristics of the } \\
\text { Sample }\end{array}$ & Interventions & Duration & Primary Results \\
\hline \multicolumn{7}{|l|}{ 1. Anti-cancer } \\
\hline Tong, 2016 [49] & In vitro & $\begin{array}{l}\text { Peimine; } \\
\text { Peiminine }\end{array}$ & $\begin{array}{l}\text { Human breast } \\
\text { carcinoma cells } \\
\text { MCF-7; Resistant cell } \\
\text { line MCF-7/ADM }\end{array}$ & $\begin{array}{c}\text { Peimine } 12.5-400 \mu \mathrm{g} / \mathrm{mL} ; \text { Peiminine } \\
12.5-400 \mu \mathrm{g} / \mathrm{mL} ; \text { ADM } \\
(0.78125-100 \mu \mathrm{g} / \mathrm{mL})+\text { Peimine or } \\
\text { Peiminine; Paclitaxel }(2.5-80 \mu \mathrm{g} / \mathrm{mL})+ \\
\text { Peimine or Peiminine }\end{array}$ & $48 \mathrm{~h}$ & $\begin{array}{l}\text { Both peimine and peiminine could reverse } \\
\text { the multi-drug resistant tumor resistance of } \\
\text { ADM or paclitaxel }\end{array}$ \\
\hline \multicolumn{7}{|l|}{$\begin{array}{l}\text { 2. Tracheobronchial } \\
\text { relaxation }\end{array}$} \\
\hline Chan, 2000 [17] & In vitro & $\begin{array}{l}\text { Peimine; } \\
\text { Peiminine; } \\
\text { Ebeiedine }\end{array}$ & $\begin{array}{l}\text { Rat tracheal and } \\
\text { bronchial rings }\end{array}$ & $\begin{array}{c}\text { Peimine, Peiminine, Ebeiedine, } \\
\text { Imperialine, puqietinone, Salbutamol, } \\
\text { Diphenhydramine, Codeine cumulative } \\
\text { concentrations } 1 \mathrm{nM}-100 \mu \mathrm{M} \\
\text { respectively }\end{array}$ & Immediate & $\begin{array}{l}\text { Peimine, peiminine, ebeiedine and } \\
\text { puqietinone in FTB could relax the } \\
\text { tracheobronch of rats. }\end{array}$ \\
\hline Wu et al., 2018 [56] & In vitro & Total alkaloids & $\begin{array}{l}\text { Rat tracheal and } \\
\text { bronchial rings }\end{array}$ & $\begin{array}{l}\text { Total alkaloids cumulative } \\
\text { concentrations } 0-3 \mathrm{~g} / \mathrm{mL}\end{array}$ & Immediate & $\begin{array}{l}\text { Total alkaloids in FTB were superior to } \\
\text { control in increasing the } \mathrm{pD}_{2} \text { value. }\end{array}$ \\
\hline $\begin{array}{l}\text { 3. Antitussive } \\
\text { Yan et al., } 2012 \text { [90] }\end{array}$ & In vivo & $\begin{array}{l}\text { Micro } \\
\text { powders }\end{array}$ & $\begin{array}{l}44 \text { guinea pigs (citric } \\
\text { acid induced cough } \\
\text { model) }\end{array}$ & $\begin{array}{l}\text { Blank control: purified water, ig,qd; } \\
\text { AZTB: } 1.5 \mathrm{~g} / \mathrm{kg} \text {, ig, qd; FTB: } 1.5 \mathrm{~g} / \mathrm{kg} \text {, ig, } \\
\text { qd; Codeine phosphate: } 0.02 \mathrm{~g} / \mathrm{kg} \text {, ig, qd }\end{array}$ & $5 \mathrm{~min}$ & $\begin{array}{l}\text { FTB was superior to control in reduding the } \\
\text { frequency of cough and prolonging the } \\
\text { remission period. }\end{array}$ \\
\hline Yan et al., 2012 [89] & In vivo & $\begin{array}{l}\text { Micro } \\
\text { powders }\end{array}$ & $\begin{array}{l}53 \text { guinea pigs (citric } \\
\text { acid induced cough } \\
\text { model) }\end{array}$ & $\begin{array}{l}\text { Blank control: purified water, ig,qd; FTB: } \\
1.5 \mathrm{~g} / \mathrm{kg}, \mathrm{ig}, \mathrm{qd} \text {; } \mathrm{AZTB}: 1.5 \mathrm{~g} / \mathrm{kg} \text {, ig, qd; } \\
\text { WBBM: } 1.5 \mathrm{~g} / \mathrm{kg}, \mathrm{ig}, \mathrm{qd} \text {; Codeine } \\
\text { phosphate: } 0.02 \mathrm{~g} / \mathrm{kg} \text {, ig, qd }\end{array}$ & $5 \mathrm{~min}$ & $\begin{array}{l}\text { FTB was superior to control in reduding the } \\
\text { frequency of cough and prolonging the } \\
\text { remission period. }\end{array}$ \\
\hline Guo, 2007 [27] & In vivo & $\begin{array}{l}\text { Aqueous } \\
\text { extract }\end{array}$ & $\begin{array}{l}140 \text { Kunming mice } \\
\text { (ammonium } \\
\text { hydroxide induced } \\
\text { cough model) }\end{array}$ & $\begin{array}{l}\text { Blank control: NS, ig,qd; FTB: 0.026, } \\
0.052,0.104 \mathrm{~g} / \mathrm{kg} \text { respectively, ig, qd; } \\
\text { Codeine phosphate: } 0.03 \mathrm{~g} / \mathrm{kg} \text {, ig, qd }\end{array}$ & 3 days & $\begin{array}{l}\text { FTB harvested in all four places was } \\
\text { superior to control in reduding the } \\
\text { frequency of cough and prolonging the } \\
\text { remission period. }\end{array}$ \\
\hline
\end{tabular}


Table 2. Cont.

\begin{tabular}{|c|c|c|c|c|c|c|}
\hline $\begin{array}{l}\text { Pharmacological } \\
\text { Effects/ } \\
\text { Included Studies }\end{array}$ & Study Type & Extract & $\begin{array}{l}\text { Characteristics of the } \\
\text { Sample }\end{array}$ & Interventions & Duration & Primary Results \\
\hline \multicolumn{7}{|l|}{ 4. Expectorant } \\
\hline Yan et al., 2012 [89] & In vivo & Fine powders & 40 mice & $\begin{array}{l}\text { Blank control: purified water, ig,qd; FTB: } \\
2 \mathrm{~g} / \mathrm{kg} \text {, ig, qd; AZTB: } 2 \mathrm{~g} / \mathrm{kg} \text {, ig, qd; } \\
\text { WBBM: } 2 \mathrm{~g} / \mathrm{kg} \text {, ig, qd; Ammonium } \\
\text { chloride: } 1 \mathrm{~g} / \mathrm{kg} \text {, ig, qd }\end{array}$ & 5 days & $\begin{array}{l}\text { Fine powders of FTB was superior to control } \\
\text { in reducing the amount of phlegm secretion. }\end{array}$ \\
\hline Wang et al., 1993 [86] & In vivo & $\begin{array}{l}\text { Alcohol } \\
\text { extract }\end{array}$ & 40 Wistar rats & $\begin{array}{l}\text { Control: starch paste, } 15 \mathrm{~g} / \mathrm{kg} \text {, ig,qd; } \\
\text { FTB: } 15 \mathrm{~g} / \mathrm{kg} \text {, ig, qd; CBM: } 15 \mathrm{~g} / \mathrm{kg} \text {, ig, } \\
\text { qd; WBM: } 15 \mathrm{~g} / \mathrm{kg} \text {, ig, qd }\end{array}$ & $5 \mathrm{~h}$ & $\begin{array}{l}\text { Alcohol extract of FTB was superior to } \\
\text { control in reducing the amount of phlegm } \\
\text { secretion. }\end{array}$ \\
\hline \multicolumn{7}{|l|}{ 5. Anti-inflammation } \\
\hline Kim et al., 2016 [33] & In vitro & $\begin{array}{c}\text { Peimine; } \\
\text { Ebeiedine; } \\
\text { Suchengbeisine }\end{array}$ & $\begin{array}{l}\text { Human } \\
\text { mucoepidermoid } \\
\text { carcinoma cells } \\
\text { NCI-H292 } \\
\end{array}$ & Peimine; Ebeiedine; Suchengbeisine & $24 \mathrm{~h}$ & $\begin{array}{l}\text { Peimine, ebeiedine, or suchengbeisine was } \\
\text { superior to control in decreasing the } \\
\text { expression of MUC5AC mucin gene. }\end{array}$ \\
\hline Zhou et al., 2017 [73] & In vitro & $\begin{array}{l}\text { Puqiedine; } \\
\text { Zhebeiresinol, } \\
\text { 2-monopalmitin, } \\
\text { N-demethylpuqie } \\
\text { Isoverticine }\end{array}$ & $\begin{array}{l}\text { Human embryonic } \\
\text { kidney cells HEK293 } \\
\text { etinone, }\end{array}$ & $\begin{array}{l}\text { FTB } 10,3,1 \mathrm{mg} / \mathrm{mL} \text { respectively; } \\
\text { Dexamethasone } 10^{-5} \mathrm{~mol} / \mathrm{L}\end{array}$ & $6 \mathrm{~h}$ & $\begin{array}{l}\text { Puqiedine, zhebeiresinol, 2-monopalmitin, } \\
\mathrm{n} \text {-demethylpuqietinone or isoverticine was } \\
\text { superior to control in reducing the } \\
\text { expression of NF-Kb level in cells. }\end{array}$ \\
\hline Xia et al., 2011 [87] & In vivo & $\begin{array}{l}\text { Aqueous } \\
\text { extract }\end{array}$ & $\begin{array}{l}30 \text { mice }(\mathrm{CP} / \mathrm{CPPS} \\
\text { model })\end{array}$ & $\begin{array}{l}\text { Blank control and blank normal: NS; } \\
\text { FTB } 0.1 \mathrm{~mL} / 10 \mathrm{~g} \text {, ig, qd }\end{array}$ & 7 days & $\begin{array}{l}\text { Aqueous extract of FTB was superior to } \\
\text { control in relieving the inflammation and } \\
\text { over proliferation of fibroblasts of the } \\
\text { prostate and reducing the serum level of } \\
\text { nitric oxide in mice. }\end{array}$ \\
\hline \multirow[t]{2}{*}{ Zhang et al., 1998 [93] } & In vivo & $\begin{array}{l}\text { Alcohol } \\
\text { extract }\end{array}$ & $\begin{array}{l}40 \text { ICR mice (xylene } \\
\text { induced ear swelling) }\end{array}$ & $\begin{array}{l}\text { Blank control: Purified water, ig,qd; } \\
\text { Ethenzamide } 0.3 \mathrm{~g} / \mathrm{kg} \text {, ig,qd; FTB 0.8, } \\
2.4 \mathrm{~g} / \mathrm{kg} \text { respectively, ig, qd }\end{array}$ & $4 \mathrm{~h}$ & $\begin{array}{l}\text { Alcohol extract of FTB was superior to } \\
\text { control in releving the swelling of ear. }\end{array}$ \\
\hline & In vivo & $\begin{array}{l}\text { Alcohol } \\
\text { extract }\end{array}$ & $\begin{array}{l}40 \text { ICR mice } \\
\text { (carrageenin induced } \\
\text { foot plantar swelling) }\end{array}$ & $\begin{array}{l}\text { Blank control: Purified water, ig,qd; } \\
\text { Ethenzamide } 0.3 \mathrm{~g} / \mathrm{kg} \text {, ig,qd; FTB 0.8, } \\
2.4 \mathrm{~g} / \mathrm{kg} \text { respectively, ig, qd }\end{array}$ & $6 \mathrm{~h}$ & $\begin{array}{l}\text { Alcohol extract of FTB was superior to } \\
\text { control in releving the swelling of foot } \\
\text { plantar. }\end{array}$ \\
\hline Xu et al., 2016 [88] & In vitro & Peimine & $\begin{array}{l}\text { Human Embryonic } \\
\text { Kidney Cells HEK293 }\end{array}$ & $\begin{array}{l}\text { Peimine concentrations } 1,310,30,100, \\
300 \mu \mathrm{M}\end{array}$ & $300 \mathrm{~s}$ & Peimine could inhibit the Nav 1.3 channel. \\
\hline
\end{tabular}


Table 2. Cont.

\begin{tabular}{|c|c|c|c|c|c|c|}
\hline $\begin{array}{l}\text { Pharmacological } \\
\text { Effects/ } \\
\text { Included Studies }\end{array}$ & Study Type & Extract & $\begin{array}{l}\text { Characteristics of the } \\
\text { Sample }\end{array}$ & Interventions & Duration & Primary Results \\
\hline \multicolumn{7}{|l|}{ 6. Pain suppression } \\
\hline \multirow[t]{2}{*}{ Zhang et al., 1998 [92] } & In vivo & $\begin{array}{l}\text { Alcohol } \\
\text { extract }\end{array}$ & $\begin{array}{l}44 \mathrm{ICR} \text { mice (acetic } \\
\text { acid induced pain) }\end{array}$ & $\begin{array}{l}\text { Blank control: Purified water, ig,qd; } \\
\text { Ethenzamide } 0.3 \mathrm{~g} / \mathrm{kg} \text {, ig,qd; FTB } 0.8, \\
2.4 \mathrm{~g} / \mathrm{kg} \text { respectively, ig, qd }\end{array}$ & $8 \mathrm{~h}$ & $\begin{array}{l}\text { Alcohol extract of FTB was superior to } \\
\text { control in reducing the frequency of mouse } \\
\text { writhing. }\end{array}$ \\
\hline & & & $\begin{array}{l}40 \text { ICR mice (thermal } \\
\text { stimulus pain) }\end{array}$ & $\begin{array}{l}\text { Blank control: Purified water, ig,qd; } \\
\text { Ethenzamide } 0.3 \mathrm{~g} / \mathrm{kg} \text {, ig,qd; FTB } 0.8 \text {, } \\
2.4 \mathrm{~g} / \mathrm{kg} \text { respectively, ig, qd }\end{array}$ & $3 \mathrm{~h}$ & $\begin{array}{l}\text { Alcohol extract of FTB was superior to } \\
\text { control in increasing the remission period of } \\
\text { tail-flick latency. }\end{array}$ \\
\hline Xu et al., 2016 [88] & In vitro & Peimine & $\begin{array}{l}\text { Human Embryonic } \\
\text { Kidney Cells HEK293 }\end{array}$ & $\begin{array}{l}\text { Peimine concentrations 1, } 310,30,100 \\
300 \mu \mathrm{M}\end{array}$ & $300 \mathrm{~s}$ & Peimine could inhibit the Nav 1.7 channel. \\
\hline \multicolumn{7}{|l|}{ 7. Antioxidation } \\
\hline Ruan et al., 2016 [85] & In vitro & Total alkaloids & $\begin{array}{l}\text { DPPH radical; ABTS } \\
\text { radical; FRAP reagent }\end{array}$ & $\begin{array}{l}\text { DPPH: Total alkaloids } 50 \mu \mathrm{L} \text {; ABTS: } \\
\text { Total alkaloids } 50 \mu \mathrm{L} \text {; FRAP: Total } \\
\text { alkaloids } 20 \mu \mathrm{L} \text {, control ethanol } 20 \mu \mathrm{L}\end{array}$ & $\begin{array}{l}\text { DPPH: } 30 \text { min; } \\
\text { ABTS: } \\
\text { Immediate; } \\
\text { FRAP: } \\
\text { Immediate }\end{array}$ & $\begin{array}{l}\text { Total alkaloids in FTB have strong a } \\
\text { antioxidative capacity evidence by the } \\
\text { results of DPPH, ABTS and FRAP. }\end{array}$ \\
\hline Ma, 2014 [84] & In vitro & polysaccharide & DPPH radical & $\begin{array}{c}\text { polysaccharide } 0.1,0.2,0.4,0.6,0.8 \\
1 \mathrm{mg} / \mathrm{mL}\end{array}$ & $30 \mathrm{~min}$ & $\begin{array}{l}\text { Polysaccharide in FTB has strong } \\
\text { antioxidative capacity evidence by the } \\
\text { results of DPPH and absorption } \\
\text { spectroscopy test. }\end{array}$ \\
\hline \multicolumn{7}{|l|}{ 8. Antiulcer } \\
\hline \multirow[t]{2}{*}{ Zhang et al., 2018 [92] } & In vivo & $\begin{array}{l}\text { Alcohol } \\
\text { extract }\end{array}$ & $\begin{array}{l}90 \mathrm{SD} \text { rats (oral ulcer } \\
\text { model) }\end{array}$ & $\begin{array}{l}\text { Blank model: NS; FTB 4, 2, } 1 \mathrm{~g} / \mathrm{kg} \\
\text { respectively, external, qid; Gui Lin Xi } \\
\text { Gua Shuang, } 1 \mathrm{~g} / \mathrm{kg} \text {, external, qid }\end{array}$ & 6 days & $\begin{array}{l}\text { Alcohol extract of FTB was superior to } \\
\text { control in inhibiting oral ulcer. }\end{array}$ \\
\hline & In vivo & $\begin{array}{l}\text { Alcohol } \\
\text { extract }\end{array}$ & $\begin{array}{l}120 \text { ICR mice (gastric } \\
\text { ulcer model) }\end{array}$ & $\begin{array}{l}\text { Blank control: Purified water, ig,qd; } \\
\text { Mepirizole } 0.05 \mathrm{~g} / \mathrm{kg} \text {, ig,qd; FTB 0.8, } \\
2.4 \mathrm{~g} / \mathrm{kg} \text { respectively, ig, qd; }\end{array}$ & $2 \mathrm{~h}$ & $\begin{array}{l}\text { Alcohol extract of FTB was superior to } \\
\text { control in inhibiting gastric ulcer. }\end{array}$ \\
\hline
\end{tabular}


Table 2. Cont

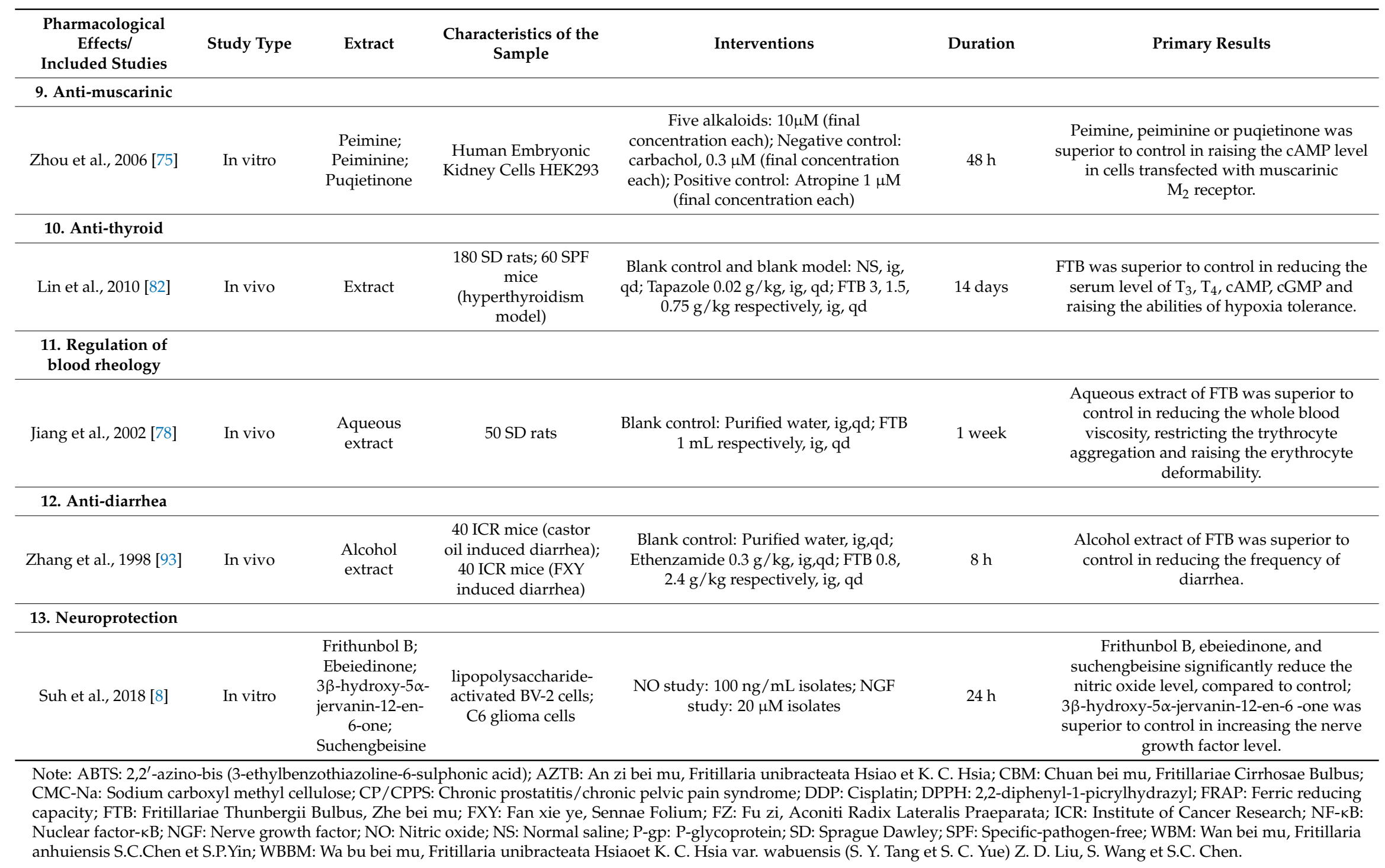




\subsubsection{Anti-Cancer Effect}

Cancer is one of the most life-threatening conditions with high morbidity and mortality in the world [102]. However, the therapeutic effects of conventional medicine are unsatisfactory, due to many adverse effects, such as fatigue, nausea and vomiting [103]. Increasingly, natural products have been identified with anti-cancer activity, which may be associated with better therapeutic effects and less adverse effects $[104,105]$. Four included studies indicated that FTB had anti-cancer effect $[9,49,80,91]$. Three of them investigated the multidrug resistance (MDR) reversal activity of FTB on human lung adenocarcinoma parental cells A549 [80], human hepatocellular carcinoma cell line HepG2 [9], and human breast carcinoma cells MCF-7 [49]. Li and colleagues reported that the total alkaloids of FTB may have the MDR reversal activity on A549/cisplatin (A549/DDP) cells [80]. Specifically, the $50 \%$ inhibitory concentration (IC50) of FTB extract in vitro was $141 \pm 5 \mathrm{mg} / \mathrm{L}$ (A549 cells) and $298 \pm 22 \mathrm{mg} / \mathrm{L}$ (A549/DDP cells) while the IC50 DDP plus FTB (9 mg/L) and DDP alone on A549/DDP cells was $14.06 \pm 3.72 \mathrm{mg} / \mathrm{L}$ and $0.79 \pm 0.14 \mathrm{mg} / \mathrm{L}$ respectively after 72 -h intervention. The reversal fold (RF) of FTB to DDP on A549/DDP cells was 17.80, higher than other reversal agents, such as cyclosporine A (10.16) and tetrandrine (14.05). The tumor inhibitory rate of DDP plus FTB $(2 \mathrm{mg} / \mathrm{kg}$ ) was $67.4 \%$, higher than DDP alone (49.9\%). The expressions of MDR1 mRNA and P-glycoprotein (P-gp) were significantly decreased by FTB extract. Similar results on the expression of P-gp were also reported by another research team in China [9]. They used the Calcein-AM Kit to identify potential P-gp inhibitors. The results showed that alkaloids and nucleoside in FTB significantly restricted the efflux activity of $\mathrm{P}$-gp in a dosage-dependent manner. Furthermore, Tong specified that peimine (A1) and peiminine (A2) were the reversal agents in FTB that reversed the MDR of adriamycin or paclitaxel in MCF7 / A cells. The RF of peimine (A1) plus adriamycin or paclitaxel and peiminine (A2) plus adriamycin or paclitaxel was $8.17,4.57,3.30$ and 3.73 respectively after 48 -h treatment. In addition, direct anti-cancer activity was also detected by in vitro and In vivo studies in one included study [91]. The researchers believed that the FTB aqueous extract $(75 \mathrm{mg} / \mathrm{mL})$ inhibited the human lung adenocarcinoma parental cells LM2 with dosage dependence and the apoptosis rate was increased over time ( $24 \mathrm{~h}: 5.7 \pm 0.91 \% ; 48 \mathrm{~h}: 11.7 \pm 1.07 \%)$. In vivo studies revealed that the tumor inhibitory rate of FTB aqueous extract $(1.6 \mathrm{~g} / \mathrm{kg})$ was $33.33 \%$. Concurrently, the same dose of FTB aqueous extract significantly reduced the number of metastases in mice $(5.7 \pm 1.72)$, compared to the control groups (7.3 \pm 2.2$)$ [91]. FTB, especially peimine (A1) and peiminine (A2), seems to have both direct and indirect anti-cancer effects evidenced by inhibiting the growth of tumor cells, as well as reversing the MDR of conventional chemotherapy drugs, such as DDP, adriamycin and paclitaxel $[9,49,80,91]$. Nonetheless, existing evidence is not sufficient to elucidate its mechanisms of actions on lung, liver or breast cancer. Therefore, more in vitro and In vivo studies are needed on lung, liver, breast and other cancers to thoroughly investigate its direct and indirect anti-cancer activity in the future.

\subsubsection{Tracheobronchial Relaxation}

Two included studies investigated the tracheobronchial relaxation activity of FTB extract in isolated bronchial and tracheal rings of rats $[17,56]$. Chan claimed that peimine (A1), peiminine (A2), ebeiedine (A5), and puqietinone (A13) extracted from FTB relaxed the tracheobronch in vitro with $6.9 \pm 0.1,6.4 \pm 0.12,6.3 \pm 0.09$ and $5.7 \pm 0.07$ bronchi $\mathrm{pD}_{2}$ values respectively and $6.9 \pm 0.12$, $6.8 \pm 0.18,6.5 \pm 0.08$ and $6.0 \pm 0.16$ tracheas $\mathrm{pD}_{2}$ values [17]. $\mathrm{pD}_{2}$ values, also called $\mathrm{pEC}_{50}$, is the negative logarithm of the half maximal effective concentration $\left(\mathrm{EC}_{50}\right)$ and if the $\mathrm{pEC}_{50}$ values are larger, the drugs are more effective [106]. Thus, the ranking order of effects within these compounds are peimine (A1) $\geq$ peiminine (A2) $>$ ebeiedine (A5) $>$ puqietinone (A13) [17]. The researcher believed that the mechanisms of action of tracheobronchial relaxation activity of FTB may be related to activation of $\mathrm{Ca}^{2+}$-activated $\mathrm{K}^{+}$channels, since peimine (A1), peiminine (A2) and ebeiedine (A5) inhibited $\mathrm{Ca}^{2+}$-induced contraction (concentration: $3 \mathrm{mM} \mathrm{Ca}^{2+}$ ) during the study [17]. Another recent study also investigated the $\mathrm{pD}_{2}$ values of FTB on tracheobronchial relaxation activity [56]. The results indicated that the total alkaloids of FTB have tracheobronchial relaxation activity with $6.66 \pm 0.06$ bronchi $\mathrm{pD}_{2}$ 
values and $6.71 \pm 0.03$ tracheas $\mathrm{pD}_{2}$ values, significantly higher than the crude water extract $(4.7 \pm 0.07$ bronchi $\mathrm{pD}_{2}$ values and $4.63 \pm 0.14$ tracheas $\mathrm{pD} 2$ values) [56]. These two studies indicated that FTB could have tracheobronchial relaxation activity, due to its alkaloids, such as peimine (A1), peiminine (A2), ebeiedine (A5) and puqietinone (A13) $[17,56]$. However, more solid evidence is required to identify the best treatment dosage of these compounds, as well as the effects of other alkaloids on tracheobronchial relaxation activity.

\subsubsection{Antitussive Effect}

Two In vivo studies were conducted by the same research team to identify the antitussive effect of FTB in guinea pigs $[89,90]$. Both of them indicated that the frequency of cough was significantly reduced $(15 \pm 7.6 / 5 \mathrm{~min})$ and the remission period was prolonged $(73.65 \pm 43.02 \mathrm{t} / \mathrm{s})$ to five minutes after oral administration of micro powders of FTB, compared to the purified water control group $(24.2 \pm 10.75 / 5 \mathrm{~min}$ and $46.99 \pm 12.02 \mathrm{t} / \mathrm{s}$ respectively) $[89,90]$. Another study compared the antitussive effects of FTB in mice harvested in four different places in China [27]. The results indicated that a high dose of FTB aqueous extract $(0.104 \mathrm{~g} / \mathrm{kg})$ harvested in all four different places significantly reduced the frequency of cough $(p<0.01)$ and increased the remission period $(p<0.05)$ [27]. As an important antitussive herb in ancient China, micro powders and aqueous extract of FTB appeared to have antitussive activity in experimental studies.

\subsubsection{Expectorant Effect}

Two studies described FTB's expectorant effect [86,89]. Yan and colleagues evaluated the expectorant effects of FTB fine powders via the phenol red expectoration test [89]. The results indicated that FTB fine powders significantly reduced the amount of phlegm secretion (phenol red contraction: $1.048 \pm 0.09 \mathrm{mg} / \mathrm{L}$ ), compared to the blank control group (phenol red contraction: $0.691 \pm 0.059 \mathrm{mg} / \mathrm{L}$ ) (mean difference $0.36,95 \%$ confidence interval 0.29-0.42) [89]. The other study assessed the amount of phlegm secretion using a percentage (per hour secretion before and after administration) [86]. The result showed that FTB dramatically reduced the amount of phlegm secretion (203.6\%, larger than the standard $170 \%$ which indicates effective treatment), compared to the control group (101.2\%) [86]. These two studies showed that the fine powders and alcohol extract of FTB produce the expectorant effect evidenced by decreasing the amount of phlegm secretion $[86,89]$. However, the active compounds related to this effect are still unknown. Further studies should investigate the active chemical constituents in FTB, as well as possible mechanisms of actions related to the expectorant effect.

\subsubsection{Anti-inflammation}

Anti-inflammation is one of the most essential pharmacological effects of FTB, mentioned in five included studies, three in vitro studies $[33,73,88]$ and two In vivo studies $[87,93]$. Three chemical compounds isolated from FTB, including peimine (A1), ebeiedine (A5) and suchengbeisine (A8), could reduce the expression and production of MUC5AC mucin (common mucin expressed in the airway surface epithelium) in human pulmonary mucoepidermoid NCI-H292 cells [33]. Peimine (A1) was able to block the Kv1.3 ion channel [88]. The Kv1.3 inhibitor is commonly believed to have the anti-inflammatory ability, blocking the human $\mathrm{T}$ lymphocytes-induced immune responses $[88,107,108]$. Another five chemical compounds, including isoverticine (A7), puqiedine (A15), $\mathrm{N}$-demethylpuqietinone (A19), 2-monopalmitin (I2), and zhebeiresinol (J2) reduced the expression of NF-kB level in the human embryonic kidney cells HEK293 [73]. In vivo studies revealed that the aqueous extract of FTB inhibited the abnormal immune response by relieving the inflammation and over the proliferation of fibroblasts of the prostate, as well as reducing the serum level of $\mathrm{NO}$ in the mice model with immunological chronic prostatitis/chronic pelvic pain syndrome [87]. Two animal studies reported that the alcohol extract of FTB relieved the swelling of ear and foot plantar induced by xylene and carrageenin respectively in mice [93]. With its anti-inflammatory 
activities, FTB could be used for many inflammatory disorders, such as pulmonary or tracheobronchial inflammatory disorders, lump or masses $[87,93]$. Future studies should focus on mechanisms of actions of the active compounds mentioned in these publications, including peimine (A1), ebeiedine (A5) and suchengbeisine (A8), isoverticine (A7), puqiedine (A15), N-demethylpuqietinone (A19), 2-monopalmitin (I2), and zhebeiresinol (J2) for anti-inflammatory activity.

\subsubsection{Pain Suppression}

FTB was found to be a potential pain reliever in two included studies [88,92]. Peimine (A1) extracted from FTB inhibited the Nav1.7 ion channel, which could be a possible mechanism of action of pain suppression [88]. Nav1.7, commonly found in the peripheral nervous system, is one number of the sodium channel family encoded by the SCN9A gene [109-111]. Blocking this channel may lead to inherited gene mutations and subsequently lead to pain sensory loss [112]. The alcohol extract of FTB reduced the frequency of mouse writhing (acetic acid induced pain test) and prolonged the remission period of tail-flick latency (thermal stimulus pain test) in mice [92]. The in vitro and in vivo studies mentioned above presented some evidence relevant to the pain suppressive activity of FTB. However, due to limited evidence, it is difficult to thoroughly explain the mechanisms of this action. Further studies could consider using peimine (A1) as an active compound for pain relief in in vitro and in vivo studies.

\subsubsection{Antioxidation}

Recent studies indicated that many herbs, including FTB have an antioxidative effect $[84,85]$. The total alkaloids extracted from FTB has strong antioxidative capacity that was reflected in the data of 2,2-diphenyl-1-picrylhydrazyl (DPPH) radical scavenging activity $\left(\mathrm{EC}_{50}: 5.5 \mathrm{mg} / \mathrm{mL}\right), 2,2^{\prime}$-azino-bis (3-ethylbenzothiazoline-6-sulphonic acid) radical scavenging activity $\left(\mathrm{EC}_{50}: 0.3 \mathrm{mg} / \mathrm{mL}\right.$ ) and ferric reducing capacity test (118.2 $\mathrm{mg}$ ascorbic acid equivalent per $100 \mathrm{~g}$ ) [85]. The polysaccharide extracted from FTB also has an antioxidative effect [84]. Specifically, the DPPH scavenging ratio of FTB was $21.46 \%$. The reducing capacity and total antioxidative capacity of FTB polysaccharide $(1 \mathrm{mg} / \mathrm{mL})$ were evaluated using the absorption spectroscopy method $(700 \mathrm{~nm})$ with 0.51 and 0.39 absorbance respectively [84]. Notwithstanding the fact that both of the in vitro studies indicated that not only total alkaloids, but also polysaccharide in FTB have a strong antioxidative capacity, in vivo studies are needed to support in vitro findings in future. In addition, identifying the active compounds related to this pharmacological effect is required.

\subsubsection{Other Pharmacological Effects}

FTB also had other pharmacological effects, including anti-ulcer, anti-muscarinic, anti-thyroid, regulation of blood rheological properties, anti-diarrhea and neuroprotection [8,75,78,82,92-94]. The alcohol extract of FTB could directly inhibit water immersion-induced or hydrochloric acid-induced gastric ulcer [92], as well as an acetic acid-induced oral ulcer in rats [94]. Three alkaloids, including peimine (A1), peiminine (A2) and puqietinone (A13), could significantly increase the cAMP level in the HEK293 cells transfected with muscarinic M2 receptor [75]. Anti-thyroid activity was detected, evidenced by reduced the serum levels of $\mathrm{T}_{3}, \mathrm{~T}_{4}, \mathrm{cAMP}$ and cGMP, and increasing hypoxia tolerance in hyperthyroidic mice [82]. FTB was able to decrease whole blood viscosity, inhibit erythrocyte aggregation and increase erythrocyte deformability in rats, which means it could regulate blood rheological properties [78]. In addition, the alcohol extract of FTB significantly decreased the frequency of diarrhea induced by castor oil or Fan xie ye (Folium Sennae) [93]. The NO level in lipopolysaccharide-activated BV-2 cells was inhibited by ebeiedinone (A6) $\left(\mathrm{IC}_{50}: 11.45 \mu \mathrm{M}\right)$, suchengbeisine (A8) (IC50: $18.02 \mu \mathrm{M})$ and frithunbol B (A24) ( $\left.\mathrm{IC}_{50}: 16.35 \mu \mathrm{M}\right)$ [8]. Moreover, the compound $3 \beta$-hydroxy-5 $\alpha$-jervanin-12-en-6-one (A26) significantly increased the nerve growth factor level $(134.81 \pm 3.66 \%)$ in C6 glioma cells [8]. In vivo and in vitro studies demonstrated that FTB may have a series of pharmacological effects. However, evidence for each pharmacological effect of FTB is 
limited. More in vitro and in vivo studies are needed to elucidate the possible mechanisms of actions of each effect.

\subsection{Pharmacokinetics of FTB}

Only six included studies of this review provided information on the pharmacokinetics of FTB's chemical compounds, such as peimine (A1), peiminine (A2) and peimisine (A9) $[45,49,59,76,77,83]$. The experimental study indicated that peiminine (A2), extracted by $70 \%$ or $90 \%$ ethyl alcohol, could penetrate excised rat skin using ultraviolet radiation testing method [83]. The penetration rates were similar in both concentrations of solvent. Although the penetration amount of peiminine (A2) in different concentrations of ethyl alcohol was similar and increased over time, the percutaneous permeability coefficient was limited and low in both groups. A sensitive, rapid and maneuverable method, LC-TRAP-MS, was developed to identify whether the nine alkaloids extracted from FTB could be absorbed by rats in plasma after oral administration [45]. The study claimed that six compounds, including peimine (A1), peiminine (A2), ebeiedinone (A6), isoverticine (A7), peimisine (A9), and puqiedinone (A14) were detected in plasma whereas three of them, including ebeiedine (A5), zhebeininoside (A11) and puqiedine (A15) were not. Furthermore, the parameters of pharmacokinetics of peimine (A1), peiminine (A2) and peimisine (A9) were investigated in four studies, including maximum concentration $\left(C_{\max }\right)$, elimination half-life $\left(\mathrm{T}_{1 / 2}\right)$, time to reach $\mathrm{C}_{\max }\left(\mathrm{T}_{\max }\right)$, oral clearance $(\mathrm{CL} / \mathrm{F})$, volume distribution/bioavailability $(\mathrm{V} / \mathrm{F})$, area under the concentration-time curve (AUC) from zero to $t$ and from zero to infinite time $[49,59,76,77]$. Table 3 summarizes the pharmacokinetic parameters of peimine (A1), peiminine (A2) and peimisine (A9) in these four studies.

One of these four studies compared the differences of pharmacokinetics, tissue distribution and excretion between male and female rats [77]. Table 3 shows that in this study, the index (Cmax, Tmax, AUC0-t and AUC0- $\infty$ ) of male rats was significantly higher than the female's while the CL/F and $\mathrm{V} / \mathrm{F}$ statistics were lower, which indicates that the elimination of peimine and peiminine in male rat plasma was slower than the female's $(p<0.05)$. In contrast, the results also showed that the cumulative excretion of peimine (A1) and peiminine (A2) in male rat urine were significantly higher than that in female. Additionally, peimine (A1) and peiminine (A2) were found in various tissues of rats, including liver, heart, spleen, kidney, lung, uterus, ovary, brain, testis, muscle and skin [77]. The concentrations of both alkaloids in male tissues were significantly higher than female's, except for muscle and skin. On the other hand, the pharmacokinetic parameters in Tong's study were substantially lower than those in the other three studies, and this may be due to its very low administration dose $(0.45 \mathrm{~g} / \mathrm{kg})$ [49]. Current pharmacokinetic studies of FTB are mainly focused on the absorption, distribution and excretion of peimine (A1), peiminine (A2) and some other alkaloids in rats. The examination of other chemical compounds will assist in understanding the pharmacokinetic profile of FTB. Further research should consider comprehensively investigating the pharmacokinetics of other chemical components listed in Table 1. 
Table 3. Pharmacokinetics of peimine (A1) and peiminine (A2) and peimisine (A9) in Fritillariae Thunbergii Bulbus.

\begin{tabular}{|c|c|c|c|c|c|c|c|c|c|c|c|}
\hline $\begin{array}{l}\text { Included } \\
\text { Studies }\end{array}$ & $\begin{array}{l}\text { Study } \\
\text { Type }\end{array}$ & Methods & Animals & Interventions & $\mathrm{T}_{1 / 2}(\mathrm{~h})$ & $T_{\max }(h)$ & $\mathrm{CL} / \mathrm{F}(\mathrm{L} / \mathrm{h} / \mathbf{k g})$ & V/F (L/kg) & $\mathrm{C}_{\max }(\mu \mathrm{g} / \mathrm{L})$ & $\begin{array}{l}\mathrm{AUC}_{0-\mathrm{t}} \\
(\mu \mathrm{g} \mathrm{h} / \mathrm{L})\end{array}$ & $\begin{array}{l}\mathrm{AUC}_{0-\infty} \\
(\mu \mathrm{gh} / \mathrm{L})\end{array}$ \\
\hline $\begin{array}{l}\text { Chen et al., } \\
2011 \text { [76] }\end{array}$ & In vivo & $\begin{array}{l}\text { LC-MS-MS; DAS } \\
2.0 \text { package }\end{array}$ & $\begin{array}{l}12 \text { female SD } \\
\text { rats }\end{array}$ & $\begin{array}{c}\text { Peimine, } \\
4.25 \mathrm{~g} / \mathrm{kg} \text {, ig }\end{array}$ & $4.8 \pm 0.8$ & $1.5 \pm 0.6$ & $119.6 \pm 40.1$ & $854.8 \pm 363.9$ & $43.2 \pm 5.4$ & $\mathrm{~N} / \mathrm{A}$ & $260.5 \pm 119.8$ \\
\hline \multirow{5}{*}{$\begin{array}{l}\text { Chen et al., } \\
2013 \text { [77] }\end{array}$} & \multirow{5}{*}{ In vivo } & \multirow{5}{*}{$\begin{array}{l}\text { LC-MS-MS; DAS } \\
2.0 \text { package }\end{array}$} & \multirow{3}{*}{$\begin{array}{l}6 \text { female SD } \\
\text { rats }\end{array}$} & $\begin{array}{c}\text { Peiminine, } \\
4.25 \mathrm{~g} / \mathrm{kg} \text {, ig }\end{array}$ & $6.6 \pm 3.2$ & $4.5 \pm 1.9$ & $34.1 \pm 4.8$ & $321.1 \pm 155.4$ & $57.6 \pm 23.0$ & $\mathrm{~N} / \mathrm{A}$ & $618.3 \pm 94.8$ \\
\hline & & & & $\begin{array}{c}\text { Peimine, } \\
4.25 \mathrm{~g} / \mathrm{kg} \text {, ig }\end{array}$ & $4.2 \pm 2.0$ & $1.5 \pm 0.7$ & $128.9 \pm 32.6$ & $781.3 \pm 305.6$ & $43.7 \pm 22.7$ & $214.2 \pm 84.6$ & $214.3 \pm 84.5$ \\
\hline & & & & $\begin{array}{l}\text { Peiminine, } \\
4.25 \mathrm{~g} / \mathrm{kg} \text {, ig }\end{array}$ & $3.4 \pm 1.7$ & $2.8 \pm 0.9$ & $36.3 \pm 15.8$ & $268.8 \pm 163.9$ & $64.2 \pm 40.0$ & $571.0 \pm 243.4$ & $571.1 \pm 243.9$ \\
\hline & & & \multirow[t]{2}{*}{$6 \mathrm{SD}$ male rats } & $\begin{array}{c}\text { Peimine, } \\
4.25 \mathrm{~g} / \mathrm{kg} \text {, ig }\end{array}$ & $6.2 \pm 1.9$ & $2.9 \pm 1.7$ & $41.5 \pm 20.1$ & $374.1 \pm 186.2$ & $57.6 \pm 21.6$ & $662.4 \pm 277.9$ & $665.3 \pm 213.3$ \\
\hline & & & & $\begin{array}{l}\text { Peiminine, } \\
4.25 \mathrm{~g} / \mathrm{kg} \text {, ig }\end{array}$ & $5.1 \pm 1.4$ & $3.0 \pm 1.4$ & $10.5 \pm 2.6$ & $92.2 \pm 55.1$ & $135.6 \pm 40.2$ & $1965.5 \pm 433.3$ & $1969.6 \pm 433.5$ \\
\hline \multirow{3}{*}{$\begin{array}{c}\text { Tong, } 2016 \\
\text { [49] }\end{array}$} & \multirow{3}{*}{ In vivo } & \multirow{3}{*}{$\begin{array}{l}\text { UHPLC-MS/MS; } \\
\text { DAS 2.0 package }\end{array}$} & \multirow{3}{*}{$\begin{array}{l}6 \text { SD male rats; } \\
6 \text { female SD } \\
\text { rats }\end{array}$} & $\begin{array}{c}\text { Peimine, } \\
0.45 \mathrm{~g} / \mathrm{kg} \text {, ig }\end{array}$ & $2.7 \pm 0.5$ & $0.5 \pm 0.2$ & $\mathrm{~N} / \mathrm{A}$ & $40.8 \pm 17.6$ & $3.7 \pm 0.9$ & $10.5 \pm 1.7$ & $10.6 \pm 1.7$ \\
\hline & & & & $\begin{array}{c}\text { Peiminine, } \\
0.45 \mathrm{~g} / \mathrm{kg} \text {, ig }\end{array}$ & $1.9 \pm 0.8$ & $0.7 \pm 0.2$ & $\mathrm{~N} / \mathrm{A}$ & $9.6 \pm 5.0$ & $12.6 \pm 2.0$ & $37.1 \pm 13.7$ & $37.1 \pm 13.8$ \\
\hline & & & & $\begin{array}{l}\text { Peimisine, } \\
0.45 \mathrm{~g} / \mathrm{kg} \text {, ig }\end{array}$ & $4.0 \pm 1.0$ & $0.8 \pm 0.3$ & $\mathrm{~N} / \mathrm{A}$ & $12.1 \pm 4.9$ & $1.2 \pm 0.2$ & $4.6 \pm 2.0$ & $4.7 \pm 2.1$ \\
\hline \multirow[t]{2}{*}{$\begin{array}{l}\text { Xu et al., } \\
2017 \text { [59] }\end{array}$} & \multirow[t]{2}{*}{ In vivo } & \multirow[t]{2}{*}{$\begin{array}{l}\text { UHPLC-MS/MS; } \\
\text { DAS } 2.0 \text { package }\end{array}$} & \multirow[t]{2}{*}{6 male SD rats } & $\begin{array}{l}\text { Peimine, } \\
20 \mathrm{~g} / \mathrm{kg} \text {, ig }\end{array}$ & $2.3 \pm 0.8$ & $2.8 \pm 1.4$ & $\mathrm{~N} / \mathrm{A}$ & $\mathrm{N} / \mathrm{A}$ & $74.7 \pm 18.2$ & $474.5 \pm 143.4$ & N/A \\
\hline & & & & $\begin{array}{l}\text { Peimisine, } \\
20 \mathrm{~g} / \mathrm{kg} \text {, ig }\end{array}$ & $\begin{array}{c}2.68 \pm \\
0.78\end{array}$ & $3.3 \pm 1.1$ & $\mathrm{~N} / \mathrm{A}$ & N/A & $15.1 \pm 2.0$ & $120.7 \pm 31.3$ & $\mathrm{~N} / \mathrm{A}$ \\
\hline
\end{tabular}

Note: DAS 2.0: Drug and Statistic 2.0; N/A: Not applicable; SD: Sprague Dawley. 


\subsection{Toxicity of FTB}

FTB is considered a non-toxic or low toxic herb for the past several thousands of years with a recommended dose of 5-10 g (equivalent to $83-167 \mathrm{mg} / \mathrm{kg}$ body weight) for adults [2]. Recently, three included studies were published which are relevant to the toxicity of FTB [79,81,92]. An in vivo study using 110 male Wistar rats indicated that the FTB water extract $(10 \mathrm{~g} / \mathrm{kg}$, ig, for seven days) did not increase the creatine kinase and lactate dehydrogenase levels compared to the saline group [79]. The histopathological examination in this study showed that the basic structure of heart tissue and cardiac muscle fibers were normal, compared to the saline group. This revealed that the extract of FTB did not induce cardiotoxicity in rats. Nonetheless, irregular cell nuclear arrangement and nonuniform cytoplasm staining were found in the FTB group.

Another two studies investigated the median lethal dose $\left(\mathrm{LD}_{50}\right)$ of oral intake FTB extract on 40 and 60 mice respectively via the acute toxicity test for seven [92] and 14 days [81] respectively. The results showed that the $\mathrm{LD}_{50}$ was $12.2 \pm 2.2 \mathrm{~g} / \mathrm{kg}$ [92] and $53 \mathrm{mg} / \mathrm{kg}$ [81] on mice respectively. Due to insufficient information, it is difficult to interpret the dramatic difference of the $\mathrm{LD}_{50}$ between these two studies. Acute toxic symptoms were observed, such as tremors, restlessness, drowsiness, asthma, convulsions, urinary incontinence, and tail-erecting reaction, and the cause of death could be pulmonary venous pleonaemia and respiratory failure [81,92]. A sub-chronic toxicity test was conducted on 120 rats after oral-intake of 1 or $3 \mathrm{mg} / \mathrm{kg}$ FTB extract for 26 weeks [81]. The findings indicated that the safe dose of FTB extract could be $1 \mathrm{mg} / \mathrm{kg}$ without any toxicity symptoms whereas tremor and reduction of spontaneous motor activities were found in the $3 \mathrm{mg} / \mathrm{kg}$ group. Furthermore, hematology, blood biochemistry, as well as weight and histology of rats' organ were observed without any significant changes within 26 weeks [81].

The recommended dose listed in the Pharmacopeia of the People's Republic of China seems to be safe, as the aqueous extract of FTB in recommended dose could be $0.0415-0.0835 \mathrm{mg} / \mathrm{kg}(0.05 \%$ extraction efficiency), which is approximately 10-25 times lower than $1 \mathrm{mg} / \mathrm{kg}$ [81]. Nonetheless, some challenges should not be neglected, such as the tremendous difference of $\mathrm{LD}_{50}$ in two different studies and the adverse effects found in the animal studies. Therefore, more experimental and clinical toxicity studies are needed, with a focus on the estimation of $\mathrm{LD}_{50}$, as well as the adverse effects of FTB at both low and high doses.

\section{Conclusions}

It is worth noting that this study was the first to systematically review the traditional uses, phytochemistry, pharmacodynamics, pharmacokinetics and toxicity of FTB. The crude herb FTB contains more than 100 chemical constituents in 11 categories with 13 possible pharmacological activities. Included studies revealed that these pharmacological activities may be mainly attributed to the alkaloids isolated from FTB. Its multiple compounds and effects may contribute to novel drug discovery for the management of multiple conditions. Current studies indicated that additional or even synergistic therapeutic effects could be produced, and may be superior to a single component, when combined multiple active constituents are used, because of the concurrent and selective interactions with multiple target proteins of a disease or a condition, such as malignant tumor, respiratory disorders and inflammation. Classic literature also suggested that there are multiple actions which may be performed by FTB. However, the mechanisms of actions of FTB are still not clear. The relationship between active chemical constituents and possible mechanisms needed to be systematically evaluated as well. High quality and well-designed in vivo and in vitro studies on the mechanisms of actions, pharmacokinetic and toxicity of FTB are recommended. A comprehensive and thorough investigation of the multi-target network pharmacology of FTB could provide insights into various conditions, and thus support evidence-based practice.

Supplementary Materials: Supplementary materials can be found at http://www.mdpi.com/1422-0067/20/7/ $1667 /$ s1. 
Funding: This research received no external funding.

Conflicts of Interest: The authors have no conflict of interest.

\section{References}

1. Bensky, D.; Clavey, S.; Stöger, E. Chinese Herbal Medicine. Materia Medica, 3rd ed.; Eastland Press: Seattle, WA, USA, 2015.

2. Chinese Pharmacopoeia Commission (Ed.) Pharmacopoeia of the People's Republic of China; China Medical Science and Technology Press: Beijing, China, 2015; Volume 1.

3. Li, H.; Hung, A.; Yang, A.W.H. A Classic Herbal Formula Danggui Beimu Kushen Wan for Chronic Prostatitis: From Traditional Knowledge to Scientific Exploration. Evid. Based Complement. Altern. Med. 2018, 2018, 1612948. [CrossRef]

4. Zhang, Z.; Wiseman, N.; Wilms, S. Jin Gui Yao Lue: Essential Prescriptions of the Golden Cabinet; Paradigm Publications: Taos, NM, USA, 2013.

5. Wang, C.Y.; Bai, X.Y.; Wang, C.H. Traditional Chinese medicine: A treasured natural resource of anticancer drug research and development. Am. J. Chin. Med. 2014, 42, 543-559. [CrossRef] [PubMed]

6. Jiangsu New Medical Academy (Ed.) Zhong Yao Da Ci Dian [Great Compendium of Chinese Medicines]; Shanghai Scientific and Technical Publishers: Shanghai, China, 1994.

7. State Administration of Traditional Chinese Medicine 'Chinese Materia Medica Committee' (Ed.) Zhong Hua Ben Cao [Chinese Materia Medica]; Shanghai Scientific and Technical Publishers: Shanghai, China, 1999.

8. Suh, W.S.; Lee, S.Y.; Park, J.E.; Kim, D.H.; Kim, S.; Lee, K.R. Two new steroidal alkaloids from the bulbs of fritillaria thunbergii. Heterocycles 2018, 96, 921-930. [CrossRef]

9. Liu, W.; Zou, F.; Li, D. Studies on P-glycoprotein inhibitor of multidrug tumor in Bulbus Fritillariae Thunbergii. Chin. J. Surg. Integr. Tradit. West. Med. 2015, 21, 379-382.

10. China Association of Chinese Medicine. Zhong Hua Yi Dian (Encyclopaedia of Traditional Chinese Medicine) [CD-ROM], 5th ed.; Hunan Electronic and Audio-Visual Publishing House: Changsha, China, 2015.

11. Chinese Medicine Board of Australia. Nomenclature List of Commonly Used Chinese Herbal Medicines. Available online: http:/ / www.chinesemedicineboard.gov.au/documents/default.aspx?record=WD15\% 2f18746\&dbid=AP\&chksum=Cs\%2baCFhYVrzbzL2\%2bYnhtRA\%3d\%3d (accessed on 22 January 2019).

12. Culley, T.M. Why vouchers matter in botanical research. Appl. Plant Sci. 2013, 1, 1300076. [CrossRef] [PubMed]

13. Kreiner, J.; Pang, E.; Lenon, G.B.; Yang, A.W.H. Saposhnikoviae divaricata: A phytochemical, pharmacological, and pharmacokinetic review. Chin. J. Nat. Med. 2017, 15, 255-264. [CrossRef]

14. Cai, W.; Xiong, Y.; Sheng, Z.; Xia, M.; Cui, M. Determination of eighteen trace elements in Fritillaria from different places by ICP-OES. Chin. J. Mod. Appl. Pharm. 2013, 30, 277-280.

15. Cai, X.; Zhang, Y.; Du, W.; Sheng, Z.; Yin, Z.; Fang, L.; Cai, B.; Ge, W. Determination of heavy metal and trace elements in fresh-cut Bulbus Fritillaria thunbergii slices by ICP-OES. Chin. Arch. Tradit. Chin. Med. 2014, 32, $2357-2359$.

16. Cao, Y.; Zhu, X.; Tan, L. Analysis of chemical composition by GC/MS and antimicrobial activities of essential oil from Fritillaria thunbergii Miq. J. Zhejiang Sci.-Tech. Univ. 2012, 29, 129-132.

17. Chan, S.W. Pharmacological and Chemical Investigations into Bulbus Fritillariae. Ph.D. Thesis, Chinese University of Hong Kong, Hong Kong, China, 2000.

18. Chen, X.; Li, X.; Mao, X.; Huang, H.; Miao, J.; Gao, W. Study on the effects of different drying methods on physicochemical properties, structure, and in vitro digestibility of Fritillaria thunbergii Miq. (Zhebeimu) flours. Food Bioprod. Process. 2016, 98, 266-274. [CrossRef]

19. Chen, Y.; Wang, S. $\beta$-D-glu 4-1 $\beta$-D-gal and sucrose determination of different Fritillarias by HPLC-ELSD. Lishizhen Med. Mater. Med. Res. 2012, 23, 1605-1606.

20. Chen, Z.; Zhou, X.; Zhang, Z.; Chen, W. Disscussion on the necessity of preserving the bulb of Zhebeimu (Fritillariae thunbergii bulbus) based on modern chemical analysis. Guid. J. Tradit. Chin. Med. Pharm. 2016, $22,44-46$.

21. Cheng, X.; Xiao, X.; Zhang, N.; Ma, S. Determination the contents of peimine and peiminine in Thumberg Fritillary Bulb by HPLC-MS. China Pharm. 2008, 11, 770-772. 
22. Cui, M.; Zhang, J.; Chen, S.; Jiang, H.; Zhou, H.; Ling, Q. Identification of alkaloids and flavonoids in all

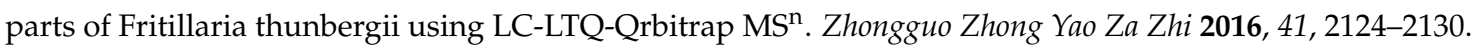
[PubMed]

23. Dai, G.; Liu, Z.; Chen, X.; Zhou, Q. Studies on the effect on the quantities of alkaloids of Bulbs Fritillariae thunbergii in different menstruum. Lishizhen Med. Mater. Med. Res. 2001, 12, 779-780.

24. Ding, X.; Li, R.; Fang, K. Contents mensuration of total alkaloid in Bulbus Fritillariae by acid dye colorinetry. China Pharma. 1999, 8, 46-47.

25. Du, W.; Zhang, H.; Yue, X.; Zhu, T.; Ge, W. The analysis on volatile components of Zhejiang Fritillary slices with different primary processing methods. Lishizhen Med. Mater. Med. Res. 2018, 29, 73-76.

26. Duan, B.; Huang, L.; Chen, S. Study on the destructive effect to inherent quality of Fritillaria thunbergii Miq. (Zhebeimu) by sulfur-fumigated process using chromatographic fingerprinting analysis. Phytomedicine 2012, 19, 562-568. [CrossRef]

27. Guo, J. Compariative Research on the Pharmaceutic and Pharmacodynamics among Different Breed Bulbus Fritillaria Thunbergii. Master's Thesis, Heilongjiang University of Chinese Medicine, Haerbin, China, 2007.

28. Huang, C. Determination of the contents of peimine A and peimine B in Fritillaria thunbergii by ELSD. China Health Stand. Manag. 2016, 7, 139-141.

29. Jiang, Y.; Li, P. Determination of verticine and verticinone in Bulbus Fritillariae Thunbergii by HPLC-ELSD. Zhongguo Yao Xue Za Zhi 2005, 40, 1257-1259.

30. Jin, X.; Xu, D.; Xu, Y.; Cui, D.; Xiao, X.; Tian, Z.; Lu, Y.; Zhen, Q. The structure identification of zhepiresionol. Acta Pharm. Sin. 1993, 28, 212-215.

31. Jin, Y.; Shen, P.; Zhang, J.; Chao, Z.; Chao, X.; Yan, Y. Separation and purification of peimine and peiminine from Bulbus Fritillariae Thunbergi by high speed countercurrent chromatography. Res. Inf. Tradit. Chin. Med. 2005, 7, 13-15.

32. Jin, Y.; Zeng, G.; Wang, L.; Wang, Z. Effects of different soaking time on extraction of active components of Fritillary Bulb. China Pharm. 2012, 21, 24-25.

33. Kim, E.J.; Yoon, Y.P.; Woo, K.W.; Kim, J.H.; Min, S.Y.; Lee, H.J.; Lee, S.K.; Hong, J.H.; Lee, K.R.; Lee, C.J. Verticine, ebeiedine and suchengbeisine isolated from the bulbs of Fritillaria thunbergii Miq. inhibited the gene expression and production of MUC5AC mucin from human airway epithelial cells. Phytomedicine 2016, 23, 95-104. [CrossRef] [PubMed]

34. Kitajima, J.; Komori, T.; Kawasaki, T. Studies on the constituents of the crude drug "Fritillariae bulbus." III. On the diterpenoid constituents of fresh bulbs of Fritillaria thunbergii Miq. Chem. Pharm. Bull. 1982, 30, 3912-3921. [CrossRef]

35. Kitajima, J.; Noda, N.; Ida, Y.; Komori, T.; Kawasaki, T. Studies on the constituents of the crude drug "Fritillariae bulbus." IV. On the diterpenoid constituents of the crudr "Fritillariae bulbus". Chem. Pharm. Bull. 1982, 30, 3922-3931. [CrossRef]

36. Li, S.L.; Lin, G.; Chan, S.W.; Li, P. Determination of the major isosteroidal alkaloids in bulbs of Fritillaria by high-performance liquid chromatography coupled with evaporative light scattering detection. J. Chromatogr. A 2001, 909, 207-214. [CrossRef]

37. Li, S.-L.; Li, P.; Lin, G.; Chan, S.-W.; Ho, Y.-P. Simultaneous determination of seven major isosteroidal alkaloids in bulbs of Fritillaria by gas chromatography. J. Chromatogr. A 2000, 873, 221-228. [CrossRef]

38. Li, W.; Bi, K.; Qian, Y.; Ning, L.; Luo, X. A pre-column derivatization HPLC method for the determination of peimine and peiminine in Bulbus Fritillariae. J. Chin. Pharm. Sci. 1996, 5, 213-218.

39. Liu, B.; Shi, R.; Lu, Y.; Zhou, Y. Study on the contents mensuration of total alkaloid in Bulbus Fritillariae by acid dye colorinetry. In Proceedings of the Fifth National Academic Conference on the Identification of Traditional Chinese Medicine, Weihai, China, 1 August 2001; pp. 152-155.

40. Liu, J.; Wang, X.; Xiang, Z.; Li, Y. Study on the principal component analysis method to determination of trace elements of Fritillaria thunbergii Miq. Guangdong Trace Elem. Sci. 2008, 15, 31-35.

41. Liu, L.; You, W.; Zheng, L.; Chen, F.; Jia, Z. Determination of peimine and peiminine in Bulbus Fritillariae Thunbergii by capillary electrophoresis by indirect UV detection using $N$-(1-naphthyl)ethylenediamine dihydrochloride as probe. Electrophoresis 2012, 33, 2152-2158. [CrossRef] [PubMed]

42. Long, Z.; Guo, Z.; Acworth, I.N.; Liu, X.; Jin, Y.; Liu, X.; Liu, L.; Liang, L. A non-derivative method for the quantitative analysis of isosteroidal alkaloids from Fritillaria by high performance liquid chromatography combined with charged aerosol detection. Talanta 2016, 151, 239-244. [CrossRef] [PubMed] 
43. Lou, Y.; Cai, H.; Liu, X.; Tu, S.; Pei, K.; Zhao, Y.; Cao, G.; Li, S.; Qin, K.; Cai, B. Element analysis and characteristic identification of non-fumigated and sulfur-fumigated Fritillaria thunbergii Miq. using microwave digestion-inductively coupled plasma atomic emission spectrometry combined with Fourier transform infrared spectrometry. Pharmacogn. Mag. 2014, 10, S30-S36. [CrossRef]

44. Ma, W.; Rong, J.; Sheng, Z.; Hu, J. Study on HPLC specific chromatogram of Fritillaric thunbergii. Chin. J. Hosp. Pharm. 2008, 28, 1458-1460.

45. Ma, Y.; Wen, Q.; Tong, X.; Fan, B.; Sun, J. Study on serum pharmacochemistry of Fritillaria thunbergii Miq. by LC-MS in rats. Chin. J. Pharmacovigil. 2014, 11, 714-720.

46. Morimoto, H.; Kimata, S. Studies on the components of Fritillaria Thunbergii Miq. I. isolation of peimine and its new glycoside. Chem. Pharm. Bull. 1960, 8, 302-307. [CrossRef]

47. Qin, J.; Chen, Y.; Luo, M.; Zheng, X.; Deng, G.; Lin, H. Comparison of in vitro dissolution rates of three constituents in superfine powder, coarse powder and decoction pieces of Fritillaria thunbergii. Chin. Tradit. Patent Med. 2017, 39, 1232-1235.

48. Que, L.; Lin, Z. Determination and comparision of alkaloids in Dongbeimu, Zhebeimu and Chuanbeimu by thin layer chromatography. Zhejiang Pharm. 1984, 1, 18-20.

49. Tong, X. Reversing Multi-Drug Resistance on Tumor Cells and Pharmacokinetics Study on Ingredients in a Traditional Chinese Medicine Fritillaria thunbergii Miq. Master's Thesis, Yunnan University of Traditional Chinese Medicine, Kunming, China, 2016.

50. Wang, A.; Hu, M. Cotents mensuration of total alkaloid in Bulbus Fritillariae by acid dye two-phase titration. Chin. J. Mod. Appl. Pharm. 1998, 15, 50-51.

51. Wang, L.; Xia, J. Determination of the proportion of peimine in Thunbery Fritillary Bulb. Heilongjiang Sci. Technol. Inf. 2011, 34. [CrossRef]

52. Wang, X.; Yang, K.; Du, L. Determination of the proportion of $\mathrm{Cu}, \mathrm{Fe}, \mathrm{Zn}$, and Ca elements in Thunbery Fritillary Bulb and Tendrilleaf Fritillary Bulb. Sichuang Food Ferment. 2007, 43, 65-67.

53. Wang, Y.; Zheng, Y. The measurement and analysis of eight elements in several sorts of Fritillaria of traditional Chinese medicine. Stud. Trace Elem. Health 2004, 21, 30-33.

54. Wu, D.; Wang, H.; Shen, P. Determination of selenium with different valences in Zhebeimu by spectrophotometry. Guangdong Trace Elem. Sci. 1998, 5, 66-68.

55. Wu, J.; Zou, Y.; Zhan, X.; Chen, S.; Lu, G.; Lai, F. Survey of heavy metal pollution in four chinese crude drugs and their cultivated soils. Bull. Environ. Contam. Toxicol. 2008, 81, 571-573. [CrossRef] [PubMed]

56. Wu, X.; Chan, S.W.; Ma, J.; Li, P.; Shaw, P.C.; Lin, G. Investigation of association of chemical profiles with the tracheobronchial relaxant activity of Chinese medicinal herb Beimu derived from various Fritillaria species. J. Ethnopharmacol. 2018, 210, 39-46. [CrossRef]

57. Wu, Z.; Li, Q. Comparative analysis of the effective components (alkaloids) of Fritillaria anhuiensis S.C.Chen et S.P.Yin, Fritillariae Thunbergii Bulbus and Fritillariae Cirrhosae Bulbus. Chin. Tradit. Herbal Drugs 1993, 24, 351-353.

58. Wu, Z.; Zheng, S. Grey relational analysis and determination of elements in Fritillaria Thunbergii Bulb and Fritillariae Cirrhosae Bulb. J. Chin. Med. Mater. 1997, 20, 291-293.

59. Xu, Y.; Li, Y.; Zhang, P.; Yang, B.; Wu, H.; Guo, X.; Li, Y.; Zhang, Y. Sensitive UHPLC-MS/MS quantitation and pharmacokinetic comparisons of multiple alkaloids from Fuzi-Beimu and single herb aqueous extracts following oral delivery in rats. J. Chromatogr. B 2017, 1058, 24-31. [CrossRef] [PubMed]

60. Xue, Y.; Gu, H. Determination of peimine and peiminine in Fritillaria thunbergii by HPLC-ELSD. Acta Pharm. Sin. 2005, 40, 550-552.

61. Yu, R.; Cheng, Z.; Gong, X. Determination and comparison of trace elements in four sort of Bulbus Fritillariae by microwave digestion-atomic absorption spectrometry. Spectrosc. Spectr. Anal. 2007, 27, 2591-2594.

62. Yuan, X.; Shi, J.; Yang, Y.; Luan, J.; Gao, J.; Wang, Y. Establishment of element fingerprint and multielement analysis of Fritillaria thunbergii by inductively coupled plasma optical emission spectrometry. Biol. Trace Elem. Res. 2010, 135, 304-313. [CrossRef] [PubMed]

63. Zeng, R.; Liu, L.; Chen, L.; Zhao, Y.; Yi, W. Study on the determination of peimine in Fritillaria thunbergii Miq from different sources by using HPLC-ELSD. J. Jaingxi Univ. Tradit. Chin. Med. 2009, 21, 51-52.

64. Zhang, C.; Sun, L.; Chen, R.; Su, J.H.; Zhang, H.F.; Gu, B.R.; Xing, Y.W.; Xue, M. Multiple analytical methods for identification and quality evaluation of Fritillariae Thunbergii Bulbus based on biological single molecules by high-performance liquid chromatography. J. Sep. Sci. 2016, 39, 3536-3543. [CrossRef] 
65. Zhang, J.; Lao, A.; Huang, H.; Ma, G.; Xu, R. Studies on chemical constituents of Fritillaria thunbergii Miq. III. isolation and identification of Zhebeinone. Acta Pharm. Sin. 1992, 27, 472-475.

66. Zhang, J.; Lao, A.; Ma, G.; Xu, R. Studies on chemical constituents of Fritillaria thunbergii Miq. II. Acta Pharm. Sin. 1991, 33, 923-926.

67. Zhang, J.; Lao, A.; Xu, R. Studies on chemical constituents of Fritillaria thunbergii Miq. IV. Chin. Bull. Bot. 1993, 35, 238-241.

68. Zhang, J.; Lao, A.; Xu, R. Studies on the chemical constituents of fresh bulbs of Fritillaria thunbergii Miq. China J. Chin. Mater. Med. 1993, 18, 354-355.

69. Zhang, J.; Ma, G.; Lao, A.; Xu, R. Studies on chemical constituents of Fritillaria thunbergii Miq. Acta Pharm. Sin. 1991, 26, 231-233.

70. Zhang, W. Study on the Determination of Alkaloid and Nucleosides in Various Species of Fritillaria. Master's Thesis, Hunan Normal University, Changsha, China, 2008.

71. Zhang, X.; Li, F.; Wang, M. HPLC quantitative analysis of uracil and three nucleotides in Bulbus Fritillariae and the application for variety discrimination. In Proceedings of the Chinese Chemical Society: The 16th National Conference on Organic Analysis and Bioanalysis Proceeding, Neimenggu, China, 12 August 2011; p. 8.

72. Zhou, J.L.; Xin, G.Z.; Shi, Z.Q.; Ren, M.T.; Qi, L.W.; Li, H.J.; Li, P. Characterization and identification of steroidal alkaloids in Fritillaria species using liquid chromatography coupled with electrospray ionization quadrupole time-of-flight tandem mass spectrometry. J. Chromatogr. A 2010, 1217, 7109-7122. [CrossRef]

73. Zhou, M.; Ma, X.; Ding, G.; Wang, Z.; Liu, D.; Tong, Y.; Zhou, H.; Gao, J.; Hou, Y.; Jiang, M.; et al. Comparison and evaluation of antimuscarinic and anti-inflammatory effects of five Bulbus fritillariae species based on UPLC-Q/TOF integrated dual-luciferase reporter assay, PCA and ANN analysis. J. Chromatogr. B 2017, 1041-1042, 60-69. [CrossRef]

74. Zhou, Y.; Gao, X.; Wu, C.; Wu, Y. Bioaccessibility and safety assessment of trace elements from decoction of "Zhebawei" herbal medicines by in vitro digestion method. J. Trace Elem. Med. Biol. 2014, 28, 173-178. [CrossRef] [PubMed]

75. Zhou, Y.; Ji, H.; Lin, B.Q.; Jiang, Y.; Li, P. The effects of five alkaloids from Bulbus Fritillariae on the concentration of cAMP in HEK cells transfected with muscarinic $\mathrm{M}_{2}$ receptor plasmid. Am. J. Chin. Med. 2006, 34, 901-910. [CrossRef] [PubMed]

76. Chen, L.; Liu, L.; Zhu, W.; Zhang, H.; Yan, Z.; Liu, H. Comparative pharmacokinetic studies of peimine and peiminine in rat plasma by LC-MS-MS after oral administration of Fritillaria thunbergii Miq. and Fritillaria thunbergii Miq.-Glycyrrhiza uralensis Fisch. couple extract. Pharmazie 2011, 66, 684-689. [CrossRef]

77. Chen, L.-H.; Zhang, H.-M.; Guan, Z.-Y.; Zhu, W.-F.; Yi, W.-J.; Guan, Y.-M.; Wang, S.; Liu, H.-N. Sex dependent pharmacokinetics, tissue distribution and excretion of peimine and peiminine in rats assessed by liquid chromatography-tandem mass spectrometry. J. Ethnopharmacol. 2013, 145, 77-84. [CrossRef] [PubMed]

78. Jiang, W.; Yang, Y.; Li, Y. Effects of some drugs for resolving phlegm on blood rehological property in rats. J. Tradit. Chin. Med. 2002, 3, 38.

79. Li, Y.; Zhou, H.; Xie, J.; Ally, M.S.; Hou, Z.; Xu, Y.; Zhang, Y. A novel method for evaluating the cardiotoxicity of traditional Chinese medicine compatibility by using support vector machine model combined with metabonomics. Evid. Based Complement. Altern. Med. 2016, 2016, e6012761. [CrossRef]

80. Li, Z.; An, C.; Hu, K.; Zhou, K.; Duan, H.; Tang, M. Multidrug resistance reversal activity of total alkaloid from Fritillaria thunbergii on cisplatin-resistant human lung adenocarcinoma A549/DDP cells. Chin. J. Pharmacol. Toxicol. 2013, 27, 315-320.

81. Li, Z.; Qiao, Y.; Li, J.; An, C.; Hu, K.; Tang, M. Acute and sub-chronic toxicity studies of the extract of Thunberg Fritillary Bulb. Regul. Toxicol. Pharmacol. 2014, 68, 370-377. [CrossRef]

82. Lin, M.; Zhou, Z.; Wang, L. Protection effect of Fritillaria thunbergii aginst hyperthyroidism in rats and mice. China Pharm. 2010, 21, 1362-1363.

83. Lin, X.; Peng, X. Experimental study on percutaneous absorption of main alkaloids in Fritillaira Thunbergii in vitro. Asia-Pac. Tradit. Med. 2011, 7, 15-18.

84. Ma, W. Antioxidant activity of polysaccharide from Fritillaria thunbergii Bulbus in vitro. Chin. Arch. Tradit. Chin. Med. 2014, 32, 1191-1193.

85. Ruan, X.; Yang, L.; Cui, W.-X.; Zhang, M.-X.; Li, Z.-H.; Liu, B.; Wang, Q. Optimization of supercritical fluid extraction of total alkaloids, peimisine, peimine and peiminine from the Bulb of Fritillaria thunbergii Miq, and evaluation of antioxidant activities of the extracts. Materials 2016, 9, 524. [CrossRef] 
86. Wang, L.; Han, C.; Wang, P. Pharmacodynamic compariason on anti-tussive and expectorant effects between Fritillaria anhuiensis S.C.Chen et S.P.Yin, Fritillariae Cirrhosae Bulbus and Fritillariae Thunbergii Bulbus. Anhui Med. J. 1993, 14, 57-58.

87. Xia, J.; Han, L.; Zhou, X.; Wei, B. The effect of Zhejiang Fritillaria Thunbergii against immunological CP/CPPS. Chin. Arch. Tradit. Chin. Med. 2011, 28, 1023-1025.

88. Xu, J.; Zhao, W.; Pan, L.; Zhang, A.; Chen, Q.; Xu, K.; Lu, H.; Chen, Y. Peimine, a main active ingredient of Fritillaria, exhibits anti-inflammatory and pain suppression properties at the cellular level. Fitoterapia 2016, 111, 1-6. [CrossRef] [PubMed]

89. Yan, X.; Tong, Z.; Luo, Y.; Tang, L.; Wu, S.; Yang, J.; Peng, C. Pharmacodynamic compariason of Fritillaria unibracteata, Fritillaria unibracteata var. wabensis and Fritillaria thunbergii cultivated. Chin. J. Exp. Tradit. Med. Formulae 2012, 18, 244-248.

90. Yan, Z.; Luo, Y.; Li, Z.; Tang, L.; Wu, S.; Yan, X.; Peng, C. Comparative studies on antiussive effect between Fritillaria unibracteata Hisao et K. C. Hisa and Fritillaria thunbergii Miq with chemical stimulation induced cough method. Lishizhen Med. Mater. Med. Res. 2012, 23, 2522-2525.

91. Yang, Q.; Nie, S.; Weng, X.; Li, L.; Huang, L. Experiment studies on anti-tumor effect in vivo and in vitro of Aconitum carmichaelii Debx. and Fritillaria thunbergii Miq. used singly or matched. Chin. J. Exp. Tradit. Med. Formulae 2005, 11, 25-28.

92. Zhang, M.; Shen, Y.; Zhu, Z.; Wang, H.; Li, F. Study on the anti-ulcer and analgesia effects of Fritillaria thunbergii Miq. Northwest Pharm. J. 1998, 13, 208-209.

93. Zhang, M.; Shen, Y.; Zhu, Z.; Wang, H.; Ma, D. Study on the anti-inflammatory and anti-diarrhea effects of Fritillaria thunbergii Miq. Hunan Guiding J. Tradit. Chin. Med. Pharmacol. 1998, 4, 30-31.

94. Zhang, W.; Xu, T.; Wang, G. Effect of extracts from Fritillaria thunbergii on oral ulcer model in rats. Mod. Pract. Med. 2018, 30, 242-243.

95. Jian, B.; Zhang, H.; Han, C.; Liu, J. Anti-cancer activities of diterpenoids derived from euphorbia fischeriana steud. Molecules 2018, 23, 23020387. [CrossRef]

96. Rohr, M.; Oleinikov, K.; Jung, M.; Sandjo, L.P.; Opatz, T.; Erkel, G. Anti-inflammatory tetraquinane diterpenoids from a Crinipellis species. Bioorg. Med. Chem. 2017, 25, 514-522. [CrossRef]

97. Kolak, U.; Kabouche, A.; Ozturk, M.; Kabouche, Z.; Topcu, G.; Ulubelen, A. Antioxidant diterpenoids from the roots of Salvia barrelieri. Phytochem. Anal. 2009, 20, 320-327. [CrossRef]

98. Hattori, H.; Tanaka, M.; Fukushima, M.; Sasaki, T.; Matsuda, A. Nucleosides and nucleotides. 158. 1-(3-C-ethynyl-beta-D-ribo-pentofuranosyl)-cytosine, 1-(3-C-ethynyl-beta-D-ribo-pentofuranosyl)uracil, and their nucleobase analogues as new potential multifunctional antitumor nucleosides with a broad spectrum of activity. J. Med. Chem. 1996, 39, 5005-5011. [CrossRef] [PubMed]

99. Ichikawa, S.; Yamaguchi, M.; Matsuda, A. Antibacterial nucleoside natural products inhibiting phospho-murNAc-pentapeptide translocase: Chemistry and structure-activity relationship. Curr. Med. Chem. 2015, 22, 3951-3979. [CrossRef] [PubMed]

100. Ichikawa, S.; Matsuda, A. Nucleoside natural products and related analogs with potential therapeutic properties as antibacterial and antiviral agents. Expert Opin. Ther. Pat. 2007, 17, 487-498. [CrossRef]

101. Jan, A.T.; Azam, M.; Siddiqui, K.; Ali, A.; Choi, I.; Haq, Q.M. Heavy metals and human health: Mechanistic insight into toxicity and counter defense system of antioxidants. Int. J. Mol. Sci. 2015, 16, 29592-29630. [CrossRef]

102. World Health Organization. Cancer. Available online: https://www.who.int/cancer/en/ (accessed on 22 January 2019).

103. National Cancer Institute. Side Effects of Cancer Treatment. Available online: https:/ /www.cancer.gov / about-cancer/treatment/side-effects (accessed on 22 January 2019).

104. Cao, H.; Mu, Y.; Li, X.; Wang, Y.; Chen, S.; Liu, J.P. A Systematic Review of Randomized Controlled Trials on Oral Chinese Herbal Medicine for Prostate Cancer. PLoS ONE 2016, 11, e0160253. [CrossRef]

105. Lin, X.; Peng, Z.; Su, C. Potential anti-cancer activities and mechanisms of costunolide and dehydrocostuslactone. Int. J. Mol. Sci. 2015, 16, 10888-10906. [CrossRef]

106. Neubig, R.R.; Spedding, M.; Kenakin, T.; Christopoulos, A. International Union of Pharmacology Committee on Receptor Nomenclature and Drug Classification. XXXVIII. Update on terms and symbols in quantitative pharmacology. Pharmacol. Rev. 2003, 55, 597-606. [CrossRef] 
107. Koo, G.C.; Blake, J.T.; Talento, A.; Nguyen, M.; Lin, S.; Sirotina, A.; Shah, K.; Mulvany, K.; Hora, D., Jr.; Cunningham, P.; et al. Blockade of the voltage-gated potassium channel Kv1.3 inhibits immune responses in vivo. J. Immunol. 1997, 158, 5120-5128. [PubMed]

108. Li, Z.; Liu, W.H.; Han, S.; Peng, B.W.; Yin, J.; Wu, Y.L.; He, X.H.; Li, W.X. Selective inhibition of CCR7(-) effector memory $\mathrm{T}$ cell activation by a novel peptide targeting Kv1.3 channel in a rat experimental autoimmune encephalomyelitis model. J. Biol. Chem. 2012, 287, 29479-29494. [CrossRef]

109. Catterall, W.A.; Goldin, A.L.; Waxman, S.G. International Union of Pharmacology. XLVII. Nomenclature and structure-function relationships of voltage-gated sodium channels. Pharmacol. Rev. 2005, 57, 397-409. [CrossRef]

110. Raymond, C.K.; Castle, J.; Garrett-Engele, P.; Armour, C.D.; Kan, Z.; Tsinoremas, N.; Johnson, J.M. Expression of alternatively spliced sodium channel alpha-subunit genes. Unique splicing patterns are observed in dorsal root ganglia. J. Biol. Chem. 2004, 279, 46234-46241. [CrossRef] [PubMed]

111. Rush, A.M.; Dib-Hajj, S.D.; Liu, S.; Cummins, T.R.; Black, J.A.; Waxman, S.G. A single sodium channel mutation produces hyper- or hypoexcitability in different types of neurons. Proc. Natl. Acad. Sci. USA 2006, 103, 8245-8250. [CrossRef]

112. Cox, J.J.; Reimann, F.; Nicholas, A.K.; Thornton, G.; Roberts, E.; Springell, K.; Karbani, G.; Jafri, H.; Mannan, J.; Raashid, Y.; et al. An SCN9A channelopathy causes congenital inability to experience pain. Nature 2006, 444, 894-898. [CrossRef] [PubMed]

(C) 2019 by the authors. Licensee MDPI, Basel, Switzerland. This article is an open access article distributed under the terms and conditions of the Creative Commons Attribution (CC BY) license (http:/ / creativecommons.org/licenses/by/4.0/). 